\title{
On the propagation of a two-dimensional viscous density current under surface waves
}

\author{
Chiu-On $\mathrm{Ng}^{\mathrm{a})}$ and Sau-Chung Fu \\ Department of Mechanical Engineering, The University of Hong Kong, Pokfulam Road, Hong Kong
}

(Received 24 July 2001; accepted 6 December 2001)

\begin{abstract}
This study aims to develop an asymptotic theory for the slow spreading of a thin layer of viscous immiscible dense liquid on the bottom of a waterway under the combined effects of surface waves and density current. By virtue of the sharply different length and time scales (wave periodic excitation being effective at fast scales, while gravity and streaming currents at slow scales), a multiple-scale perturbation analysis is conducted. Evolution equations are deduced for the local and global profile distributions of the dense liquid layer as functions of the slow-time variables. When reflected waves are present, the balance between gravity and streaming will result, on a time scale one order of magnitude longer than the wave period, in an undulating water/liquid interface whose displacement amplitude is much smaller than the thickness of the dense liquid layer. On the global scale, the streaming current can predominate and drive the dense liquid to propagate with a distinct pattern in the direction of the surface waves. (C) 2002 American Institute of Physics.
\end{abstract}

[DOI: $10.1063 / 1.1448348]$

\section{INTRODUCTION}

Accidental spills of oil or hazardous liquid hydrocarbons in near-shore regions are now common occurrences and often lead to long-term and extensive damage to the environment. Dense oils or nonaqueous phase liquids (DNAPL) that sink in water, when spilled into a body of water, will eventually reach the bottom of the water column, where they may cause further pollution by interacting with the benthic environment. Even a floating oil, when mixed with $2 \%-3 \%$ of sand, can make itself heavier than water and sink. The potential threat to the environment posed by a hazardous liquid chemical on the bottom very often is as enormous as, if not more than, that caused by a floating oil on the water surface, which is more visible to the public.

Chemicals spilled on the sea can be dissipated by natural causes such as physical mixing, chemical weathering, and biological degradation. Such causes, however, become less effective for liquid chemicals deposited on the sea bottom. Unlike those floating on the surface, they are less subjected to dissipation due to volatilization and photo-degradation. The adverse environment on the bottom also limits the aerobic biodegradation. Turbulent mixing is suppressed if stratification is stable. It is in general difficult to monitor the spreading of a liquid phase chemical on the sea bottom. A model very often is the only readily available tool by which one can estimate the extent of pollutant migration in the benthic environment.

Gravity or density current is the mechanism by which a viscous fluid spreads under a lighter fluid; it also refers to the flow along a boundary layer of one fluid intruding into another fluid as driven by gravitational or buoyancy force. ${ }^{1}$

\footnotetext{
a) Author to whom correspondence should be addressed. Telephone: (852) 2859 2622; fax: (852) 2858 5415; electronic mail: cong@hku.hk
}

Huppert and Simpson ${ }^{2}$ reasoned that a gravity current might evolve to go through three stages. The first one is the slumping phase, during which the current is retarded by the counterflow in the fluid into which it is discharging. The next two stages are, respectively, the balance between buoyancy force and inertia force, and the balance between buoyancy force and viscous force. For spreading after a sufficiently long time, the third stage is expected to dominate. This flow regime was studied experimentally by Didden and Maxworthy ${ }^{3}$ and similarity solutions for two-dimensional and axisymmetric viscous gravity currents were obtained by Huppert. ${ }^{4}$ More recent studies on gravity current include Ungarish and Huppert ${ }^{5}$ and Hogg et al. ${ }^{6}$ However, in typical coastal situations, the migration of a dense liquid in the bottom boundary layer can be forced by gravity current as well as Eulerian streaming current induced by surface waves. In those abovementioned works the effects of surface waves are grossly ignored.

For small-amplitude periodic surface water waves, the fluid particles near the bottom possess, apart from their orbital motion, a steady second-order drift velocity, which is usually termed the mass transport velocity or streaming. The theory of streaming by surface waves was studied in detail by Longuet-Higgins. ${ }^{7}$ Carter et al. ${ }^{8}$ studied the mass transport in a homogeneous fluid under incident and reflected waves. Dalrymple and Liu ${ }^{9}$ developed a general theory for linear waves propagating in a two-layer system, with the effects of all the boundary layers taken into account. Extending this work to the second order, Sakakiyama and Bijker ${ }^{10}$ obtained the mass transport velocity in a viscous mud layer due to progressive waves. More recently, $\mathrm{Ng}^{11}$ deduced analytical solutions for an asymptotic case of Dalrymple and Liu, ${ }^{9}$ namely when the lower layer of fluid is comparable in thickness with the Stokes boundary layer. $\mathrm{Ng}^{11}$ presented explicit expressions for the wave attenuation, mass transport 
velocity, and mean discharge rate of mud. For the spreading of a dense and immiscible fluid on the sea bottom, the induced wave streaming can be as influential as the gravity current, if the fluid layer profile is slowly varying. It is of fundamental interest to find out how the two driving forces will interact or counteract with each other in controlling the transport of matter in a wave boundary layer. It is the intention of this paper to study the spreading of a submerged fluid under a balance between the viscous, gravity and oscillatory effects.

The aim of this study is to develop an asymptotic theory for the spreading of a thin slowly varying layer of dense liquid on the bottom of a nearshore waterway under the action of small-amplitude surface gravity waves. In Sec. II the problem is further defined and the assumptions are stated. The relative orders of magnitude of individual effects are estimated in terms of the small parameter of wave steepness, which is the ratio of wave amplitude to wavelength. Multiple spatial and time scales exist in the problem, thereby requiring the multiple-scale expansions of the governing equations and boundary conditions. An asymptotic analysis is performed in Sec. III to obtain perturbation equations to the second order. The flow structure at the leading order can be modeled by that of a two-layer Stokes boundary layer. ${ }^{11}$ The second-order steady current, composed of Eulerian streaming and density current, is then found analytically. The problem is closed when the evolution equations, on the local and the global scales, are determined from the depth-integrated continuity equation. Owing to reflected waves, the streaming current varies periodically in half a wavelength, leading to the development of an undulating interface between water and the dense liquid on the local scale, whose analytical form is presented in Sec. IV. It is shown that an undulation is developed such that its crest is typically under a node of the surface waves, and its amplitude is larger for a smaller density contrast or a thinner layer of the dense liquid. The local streaming current structures are examined in detail in terms of the reflection coefficient and the development stage of the undulating interface. The spreading of the dense liquid also takes place slowly on a global length scale. In Sec. V the global evolution equation, which encompasses both streaming and density currents, is solved numerically for the spreading pattern as a function of time for a given initial profile. The pattern can be rather distinct and the entire fluid layer can migrate significantly in the direction of wave propagation after a sufficiently long time. Results are examined for various values of the fluid properties and wave characteristics.

\section{ASSUMPTIONS AND FORMULATION}

Consider a two-dimensional thin layer of dense viscous and immiscible nonaqueous liquid (simply referred to as the dense liquid hereafter) lying on the bottom of a water course, as shown in Fig. 1. Axes $x$ and $y$ are, respectively, in the direction of the incident wave and vertically upward from the mean water free surface. The densities of the dense liquid and water are, respectively, denoted by $\rho_{m}$ and $\rho_{w}$, where $\rho_{m}>\rho_{w}$. The flow of water near the bottom boundary is

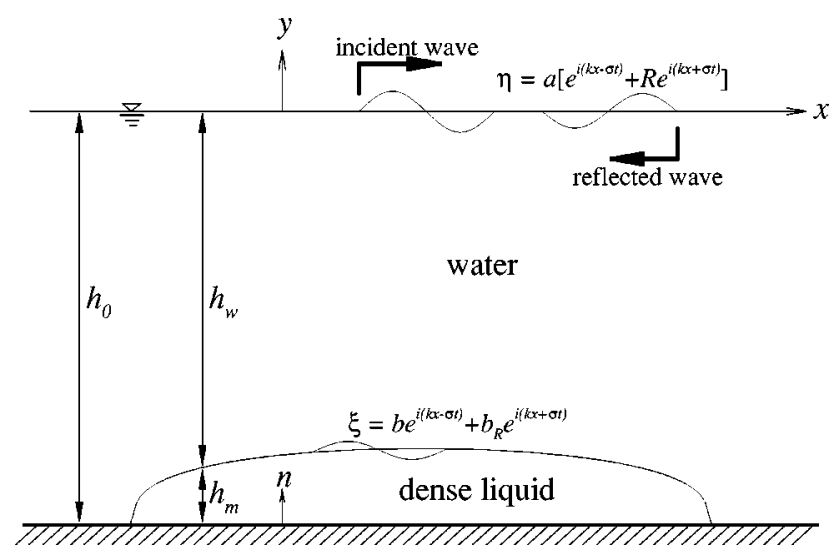

FIG. 1. Schematic diagram of the problem under consideration; a thin lens of viscous dense liquid spreading on the bottom of a waterway under surface waves.

essentially turbulent, but the dense liquid is so glutinous that its viscous motion is still largely controlled by molecular viscosity. It is assumed that stable density stratification prevails, and therefore mixing across the water/liquid interface is suppressed. Such an assumption has been commonly adopted in studies of concentrated mud under surface waves (e.g., Mei and $\mathrm{Liu}^{12}$ ). The eddy viscosity of water in the boundary layer, denoted by $\nu_{w}$, is for simplicity taken to be a constant, which in coastal zones may have typical values $1-100 \mathrm{~cm}^{2} \mathrm{~s}^{-1}$. The molecular viscosity coefficient of the dense liquid, denoted by $\nu_{m}$, also can be as much as these values if the liquid is sufficiently viscous.

The dense liquid layer is of a thickness $h_{m}(x, t)$, while the depth of the overlying water layer is $h_{w}(x, t)$; both vary slowly with the longitudinal coordinate $x$ and time $t$. The total depth $h_{0}=h_{w}+h_{m}$ is assumed to be constant, and $h_{m}$ $\ll h_{w}$. The shallowness of the dense liquid layer allows the application of the lubrication theory here.

The present theory applies to the case when a sufficiently long time has elapsed after the initial discharge of the dense liquid. In other words, it has come to a stage in which the steady fluid motion in the boundary layer that is induced by surface waves is comparable in magnitude with that driven by buoyancy. Therefore, as long as the surface waves are small in amplitude, the inertia of the fluid will be negligible at the leading order, but gives rise to a steady streaming at the next order. The steady streaming, which owes its existence to viscosity, is then balanced by gravity current. Such a buoyancy-viscous balance can also be argued in a more rigorous manner using the relations presented by Huppert. ${ }^{4}$

Now, a long-crested small-amplitude incident wave is progressing on the water free surface in the positive $x$ direction. A reflective boundary may exist farther down the water course so that a standing wave can be formed from the superposition of incident and reflected waves of the same period. The free-surface displacement can be written as

$$
\eta(x, t)=\operatorname{Re}\left[a\left\{e^{i(k x-\sigma t)}+\operatorname{Re}^{i(k x+\sigma t)}\right\}\right],
$$

where Re denotes the real part of the expression following, $a$ is the amplitude of the incident waves, $i$ is the imaginary unit, $k$ is the wave number, $\sigma$ is the wave angular frequency, 
TABLE I. Orders of magnitude and normalized forms (distinguished by a caret) of the variables.

\begin{tabular}{lll}
\hline \hline Physical variable & Order of magnitude & \multicolumn{1}{c}{ Normalized variable } \\
\hline$x, x_{1}$ & $k^{-1}$ & $\left(\hat{x}, \hat{x}_{1}\right)=k x$ \\
$x_{2}$ & $(\epsilon k)^{-1}$ & $\hat{x}_{2}=\left(k^{2} a^{2} / \delta_{m}\right) x$ \\
$n, h_{m}, h_{m 0}$ & $\left(\hat{n}, \hat{h}_{m}, \hat{h}_{m 0}\right)=\left(n, h_{m}, h_{m 0}\right) / \delta_{m}$ \\
$h_{m 1}$ & $\epsilon k^{-1}=a \sim \delta_{m}$ & $\hat{h}_{m 1}=h_{m 1} /\left(k a^{2}\right)$ \\
$\xi, b$ & $\epsilon a$ & $(\hat{\xi}, \hat{b})=(\xi, b) /\left(k \sigma^{-1} \delta_{m} \widetilde{U}_{I}\right)$ \\
$t, t_{1}$ & $\epsilon a$ & $\left(\hat{t}, \hat{t}_{1}\right)=\sigma t$ \\
$t_{2}$ & $\sigma^{-1}$ & $\hat{t}_{2}=\left(k \sigma \delta_{m}\right) t$ \\
$t_{4}$ & $(\epsilon \sigma)^{-1}$ & $\hat{t}_{4}=\left(k^{3} \sigma a^{4} / \delta_{m}\right) t$ \\
$\rho_{w}$ & $\left(\epsilon^{3} \sigma\right)^{-1}$ & $\gamma=\rho_{w} / \rho_{m}$ \\
$\delta_{w}$ & $\rho_{m}$ & $\zeta=\delta_{m} / \delta_{w}$ \\
$u, u_{0}$ & $\delta_{m}$ & $\left(\hat{u}, \hat{u}_{0}\right)=\left(u, u_{0}\right) / \tilde{U}_{I}$ \\
$v, v_{0}$ & $\sigma a \sim \widetilde{U}_{I}$ & $\left(\hat{v}, \hat{v}_{0}\right)=\left(v, v_{0}\right) /\left(k \delta_{m} \widetilde{U}_{I}\right)$ \\
$u_{1}, u_{L}$ & $\epsilon \sigma a \sim \epsilon \widetilde{U}_{I}$ & $\left(\hat{u}_{1}, \hat{u}_{L}\right)=\left(u_{1}, u_{L}\right) /\left(k \sigma^{-1} \tilde{U}_{I}^{2}\right)$ \\
$v_{1}, v_{L}$ & $\epsilon \sigma a \sim \epsilon \widetilde{U}_{I}$ & $\left(\hat{v}_{1}, \hat{v}_{L}\right)=\left(v_{1}, v_{L}\right) /\left(k^{2} \sigma^{-1} \delta_{m} \widetilde{U}_{I}^{2}\right)$ \\
$P / \rho$ & $\epsilon^{2} \sigma a \sim \epsilon^{2} \widetilde{U}_{I}$ & $\hat{P}=P /\left(\rho k^{-1} \sigma \widetilde{U}_{I}\right)$ \\
\hline \hline
\end{tabular}

and $R$ is the reflection coefficient. Without loss of generality, we may take $a$ and $\sigma$ to be real. Our focus will only be on the cases in which the coefficient $R$ is real and in the range 0 $\leqslant R \leqslant 1$. The wave is purely progressive when $R=0$, and becomes purely standing when $R=1$; a partial standing wave results from an intermediate value of $R$.

The wave steepness $\epsilon \equiv k a \ll 1$ is a small quantity, and will be used as the ordering parameter. The motion of water is essentially inviscid and irrotational except in a thin layer near the bottom in which vorticity is appreciable. Under a periodic forcing given by Eq. (1), the thin boundary layer is a Stokes boundary layer, whose thickness is typically defined as (e.g., $\mathrm{Mei}^{13}$ )

$$
\delta=(2 \nu / \sigma)^{1 / 2} .
$$

Momentum exchange renders the Stokes boundary layer to extend across the interface between the near-bottom water and the dense liquid. Since $\nu_{m} \sim \nu_{w}$, the thickness of the Stokes boundary layer in either fluid is comparable with each other. In this study, we assume that the dense liquid layer thickness $h_{m}$ and the Stokes boundary layer thickness $\delta$ are of the same order of magnitude as the wave amplitude $a$. They are supposed to be in the order of tens of centimeters, much shorter than the wavelength. Therefore, there is a sharp contrast in the horizontal and vertical length scales:

$$
\epsilon \equiv k a \sim k h_{m} \sim k \delta \ll 1 .
$$

Also, the entire dense liquid layer, as well as the near-bottom water, is subject to viscous shear. Flows in this two-layer Stokes boundary layer are describable by the classical boundary layer theory. For convenience, we introduce a local vertical coordinate $n \equiv y+h_{0}$, which points upward from the base of the dense liquid layer (Fig. 1). Note that $n$, which has the same scale as $h_{m}$, is an inner independent variable for the boundary layer solutions. In the equations and boundary conditions presented below, the small parameter $\epsilon$ is inserted merely to reflect the relative order of magnitude of the associated term, and also that of the truncation error. Otherwise, the $\epsilon$ 's may be disregarded. The parameter $\epsilon$ appears only in equations or boundary conditions which contain terms of dif- ferent orders. Such orders of the terms are obtainable upon nondimensionalizing the equations and boundary conditions using the normalized variables, which are introduced in Table I. The parameter $\epsilon$ is then retained for identification when reverting to the physical variables, thereby yielding the present equations and boundary conditions. See $\mathrm{Fu}^{14}$ for further details.

The continuity and momentum equations read as follows, where the subscript $f$ is replaced by $m$ and $w$ when the equations are applied to the dense liquid and the near-bottom water, respectively:

$\frac{\partial u_{f}}{\partial x}+\frac{\partial v_{f}}{\partial n}=0$

$$
\begin{aligned}
\frac{\partial u_{f}}{\partial t} & +\epsilon u_{f} \frac{\partial u_{f}}{\partial x}+\epsilon v_{f} \frac{\partial u_{f}}{\partial n} \\
& =-\frac{1}{\rho_{f}} \frac{\partial P_{f}}{\partial x}+\nu_{f} \frac{\partial^{2} u_{f}}{\partial n^{2}}-\frac{\rho_{f}-\rho_{w}}{\rho_{f}} g \frac{\partial h_{m}}{\partial x}+O\left(\epsilon^{2}\right),
\end{aligned}
$$

and

$$
0=-\frac{1}{\rho_{f}} \frac{\partial P_{f}}{\partial n}+O\left(\epsilon^{2}\right),
$$

where $u(x, n, t)$ and $v(x, n, t)$ are the horizontal and vertical components of the fluid velocity, and $P(x, n, t)$ is the dynamic pressure (i.e., the static pressure being subtracted from the total pressure). One may notice that in the horizontal momentum equation (5), the convective inertia are of an order $O(\epsilon)$ relative to the local acceleration, and the dense liquid is subject to buoyancy force.

At the base of the dense liquid $n=0$, the no-slip and no-leakage conditions apply:

$$
u_{m}=v_{m}=0 \text { at } n=0 .
$$

Excited by the surface waves, the interface between the dense liquid and water displaces periodically in the same manner as the surface waves. The interface is given by $F(x, n, t) \equiv n-h_{m}-\epsilon \xi=0$, where 


$$
\xi(x, t)=\operatorname{Re}\left[b e^{i(k x-\sigma t)}+b_{R} e^{i(k x+\sigma t)}\right]
$$

is the interfacial displacement with complex amplitudes $b$ and $b_{R}$ in response to the incident and reflected waves, respectively. As shown later, these amplitudes are one order of magnitude smaller than the surface wave amplitude, i.e., $b$ $=O(\epsilon a)$. The velocity and stress components are continuous at the interface $F=0$ :

$u_{w}=u_{m}, \quad v_{w}=v_{m}, \quad \boldsymbol{\tau}_{w} \cdot \nabla F=\boldsymbol{\tau}_{m} \cdot \nabla F \quad$ at $n=h_{m}+\epsilon \xi$,

where $\tau$ is the stress tensor. Using Taylor series expansion about the mean position $n=h_{m}$, and substituting the usual linear constitutive law for $\tau$, these conditions for the continuity of velocity and stress at the interface can be approximated as

$$
\begin{array}{r}
u_{w}+\epsilon \xi \frac{\partial u_{w}}{\partial n}=u_{m}+\epsilon \xi \frac{\partial u_{m}}{\partial n}+O\left(\epsilon^{2}\right) \quad \text { at } n=h_{m}, \\
v_{w}+\epsilon \xi \frac{\partial v_{w}}{\partial n}=v_{m}+\epsilon \xi \frac{\partial v_{m}}{\partial n}+O\left(\epsilon^{2}\right) \quad \text { at } n=h_{m}, \\
\rho_{w} \nu_{w}\left(\frac{\partial u_{w}}{\partial n}+\epsilon \xi \frac{\partial^{2} u_{w}}{\partial n^{2}}\right)=\rho_{m} \nu_{m}\left(\frac{\partial u_{m}}{\partial n}+\epsilon \xi \frac{\partial^{2} u_{m}}{\partial n^{2}}\right) \\
+O\left(\epsilon^{2}\right) \quad \text { at } n=h_{m},
\end{array}
$$

and

$$
\begin{aligned}
P_{w}+\epsilon\left(\xi \frac{\partial P_{w}}{\partial n}-\rho_{w} g \xi\right)= & P_{m}+\epsilon\left(\xi \frac{\partial P_{m}}{\partial n}-\rho_{m} g \xi\right) \\
& +O\left(\epsilon^{2}\right) \text { at } n=h_{m} .
\end{aligned}
$$

Also, the kinematic boundary condition on the interface is given by $d F / d t=0$, which can be expressed as

$$
\frac{\partial}{\partial t}\left(h_{m}+\epsilon \xi\right)+\epsilon u_{m} \frac{\partial}{\partial x}\left(h_{m}+\epsilon \xi\right)=\epsilon v_{m} \quad \text { at } n=h_{m}+\epsilon \xi,
$$

which for later deduction is not Taylor-series expanded at this point.

Farther up from the boundary layer but still close to the bottom, the velocity and pressure will tend to the nearbottom values given by the inviscid theory

$$
\left(u_{w}, P_{w}\right) \rightarrow\left(U_{I}, P_{I}\right)
$$

at outer edge of the boundary layer,

where $U_{I}$ and $P_{I}$ are, respectively, the horizontal component of velocity and the dynamic pressure of the inviscid flow near the bottom. The inviscid flow at this boundary is governed by

$$
\frac{\partial U_{I}}{\partial t}+\epsilon U_{I} \frac{\partial U_{I}}{\partial x}=-\frac{1}{\rho_{w}} \frac{\partial P_{I}}{\partial x} \text { near the bottom. }
$$

On the free surface, the inviscid flow is subject to the usual kinematic and dynamic boundary conditions for surface waves, which in the linearized forms are

$$
\frac{\partial \eta}{\partial t}=v_{w}+O(\epsilon) \quad \text { at } y=0
$$

$$
\frac{\partial u_{w}}{\partial t}=-g \frac{\partial \eta}{\partial x}+O(\epsilon) \text { at } y=0 .
$$

The basic mathematical formulation is now complete. The problem under consideration features several phenomena that are effective at different length and time scales. In order to prepare grounds for the ensuing asymptotic analysis, we need to establish the relationships between these scales. As discussed earlier, the basic horizontal length and time scales are, respectively, the wavelength and wave period, or $L_{1}=2 \pi k^{-1}$ and $T_{1}=2 \pi \sigma^{-1}$. The corresponding variables are the fast space and time variables $x_{1}=x$ and $t_{1}=t$.

The dense liquid layer has a thin and slowly varying profile on the $x-n$ plane. More specifically, it is assumed that

$$
\frac{\partial h_{m}}{\partial x}=O\left(\epsilon k h_{m}\right),
$$

which implies that the entire span of the dense liquid layer is much longer than the wavelength. This necessitates the introduction of a long spatial scale $L_{2}=\epsilon^{-1} L_{1} \sim\left(k^{2} a\right)^{-1}$, or a slow space variable $x_{2}=\epsilon x$. The thickness $h_{m 0}$, the leading order value of $h_{m}$, will appear constant over a wavelength and steady over a wave period, and therefore does not depend on the fast space and time variables:

$$
\frac{\partial h_{m 0}}{\partial x_{1}}=\frac{\partial h_{m 0}}{\partial t_{1}}=0 .
$$

Hence in Eqs. (5) and (14), the terms $\partial h_{m} / \partial x$ and $\partial h_{m} / \partial t$ will have no contribution until $O(\epsilon)$. The short and long length scales $\left(L_{1}, L_{2}\right)$ will also be referred to as the local and global scales.

Eulerian streaming under a standing wave varies periodically in half of a wavelength, and as a result the dense liquid layer profile may vary over this short length scale as well (see Sec. IV). This local variation in profile, in the form of periodic undulation, however, becomes effective only on a longer time scale and its magnitude is expected to be much smaller than the layer thickness itself. On balancing the gravity effect with the vertical momentum diffusion by viscosity, i.e.,

$$
\left(1-\frac{\rho_{w}}{\rho_{m}}\right) g \frac{\partial h_{m 1}}{\partial x_{1}} \sim \nu_{m} \frac{\partial^{2} \bar{u}_{m 1}}{\partial n^{2}},
$$

where $h_{m 1}$ is the amplitude of an undulation and $\bar{u}_{m 1}$ $=O\left(\sigma k a^{2}\right)$ is the second-order steady streaming velocity, one may show that $h_{m 1} \sim \epsilon h_{m}$. It is then clear from the conservation of mass

$$
\frac{\partial h_{m 1}}{\partial t} \sim \frac{\partial}{\partial x_{1}} \int \bar{u}_{m 1} d n
$$

that the time scale for the undulation development is one order of magnitude longer than the wave period. The undulations are therefore formed in $T_{2}=\epsilon^{-1} T_{1}$, and the corresponding slower time variable is $t_{2}=\epsilon t$.

The second-order streaming velocity is also responsible for the spreading and migration of the dense liquid (see Sec. 
V). Therefore, the time scale for migration of the liquid layer to be effective over a distance comparable with the length of the layer itself will be

$$
T_{4} \sim L_{2} / \bar{u}_{m 1} \sim\left(\sigma k^{3} a^{3}\right)^{-1}=O\left(\epsilon^{-3} T_{1}\right),
$$

i.e., three orders of magnitude longer than the wave period. The slowest pertinent time variable is therefore $t_{4}=\epsilon^{3} t$. The intermediate time variable $t_{3}=\epsilon^{2} t$ is later shown to be a trivial variable for the present problem, but is included for the time being for completeness.

We summarize in Table I the scalings of all the variables that are introduced above or later, and also their normalized forms, which are distinguished by a caret. Now, the multiplescale perturbation analysis can be started upon substituting into the governing equations and boundary conditions the following expansions:

$$
A \rightarrow A_{0}+\epsilon A_{1}+\epsilon^{2} A_{2}+\cdots,
$$

where $A$ stands for $u_{w}, v_{w}, u_{m}, v_{m}, U_{I}, P_{w}, P_{m}$, and $P_{I}$. The leading order quantities are further assumed to be in a first-harmonic periodic form

$$
\begin{aligned}
A_{0}= & \operatorname{Re}\left[\tilde{A}\left(x_{2}, t_{2}, t_{3}, t_{4}, n\right) e^{i\left(k x_{1}-\sigma t_{1}\right)}\right. \\
& \left.+\widetilde{A}_{R}\left(x_{2}, t_{2}, t_{3}, t_{4}, n\right) e^{i\left(k x_{1}+\sigma t_{1}\right)}\right] .
\end{aligned}
$$

The phases are functions of the fast variables, while the amplitudes depend on the slow variables only. The second term in Eq. (25) vanishes in the case of pure progressive waves. The spatial and temporal derivatives are accordingly expanded into the multiple-scale rates of change:

$$
\begin{aligned}
& \frac{\partial}{\partial x} \rightarrow \frac{\partial}{\partial x_{1}}+\epsilon \frac{\partial}{\partial x_{2}}, \\
& \frac{\partial}{\partial t} \rightarrow \frac{\partial}{\partial t_{1}}+\epsilon \frac{\partial}{\partial t_{2}}+\epsilon^{2} \frac{\partial}{\partial t_{3}}+\epsilon^{3} \frac{\partial}{\partial t_{4}} .
\end{aligned}
$$

The dense layer thickness is expanded, as discussed above, into

$$
h_{m}=h_{m 0}\left(x_{2}, t_{2}, t_{3}, t_{4}\right)+\epsilon h_{m 1}\left(x_{1}, t_{2}\right)+O\left(\epsilon^{2}\right) .
$$

Therefore, while $h_{m}$ varies slowly in both space and time at the leading order, it depends on the fast-space but slow-time variables at the next order. It will be further shown later that $h_{m 0}$ is independent of $t_{2}$ and $t_{3}$ (Sec. III C), and $h_{m 1}$ can be a periodic function of $x_{1}$ with a zero spatial mean (Sec. IV); $h_{m 1}$ also vanishes identically when $R=0$ (i.e., pure progressive waves).

\section{ASYMPTOTIC ANALYSIS}

\section{A. First-order solutions}

By linearity, the leading order solutions are a sum of components due to the incident and reflected waves [cf. Eq. (25)]. One can readily show that, for a free-surface displacement given by Eq. (1),

$$
\begin{aligned}
\left(u_{f 0}, v_{f 0}, U_{I 0}, \xi\right)= & \operatorname{Re}\left[\left(\tilde{u}_{f}, \tilde{v}_{f}, \tilde{U}_{I}, b\right) e^{i\left(k x_{1}-\sigma t_{1}\right)}\right. \\
& +R\left(-\tilde{u}_{f}^{*}, \tilde{v}_{f}^{*},-\tilde{U}_{I}^{*}, b^{*}\right) \\
& \left.\times e^{i\left(k x_{1}+\sigma t_{1}\right)}\right] \text { for } f=m, w,
\end{aligned}
$$

where $R$ is the reflection coefficient and the asterisk denotes the complex conjugate. Therefore, as shown above, the amplitudes of the quantities associated with the reflected wave are related to their counterparts associated with the incident wave, and it suffices to present below the solutions for pure progressive waves, which have been developed by $\mathrm{Ng} .^{11}$

$$
\begin{aligned}
& \text { In } 0 \leqslant n \leqslant h_{m}, \\
& \tilde{u}_{m}(n)=\left[\gamma-\gamma \cosh \lambda_{m} n+E \sinh \lambda_{m} n\right] \tilde{U}_{I}
\end{aligned}
$$

and

$$
\begin{aligned}
\tilde{v}_{m}(n)= & -i k \lambda_{m}^{-1}\left[\gamma\left(\lambda_{m} n-\sinh \lambda_{m} n\right)\right. \\
& \left.+E\left(\cosh \lambda_{m} n-1\right)\right] \tilde{U}_{I} .
\end{aligned}
$$

In $h_{m} \leqslant n<\infty$,

$$
\tilde{u}_{w}(n)=\left[1+D e^{-\lambda_{w}\left(n-h_{m}\right)}\right] \tilde{U}_{I}
$$

and

$$
\begin{aligned}
\tilde{v}_{w}(n)= & \left.\widetilde{v}_{m}\right|_{n=h_{m}}-i\left[k\left(n-h_{m}\right)\right. \\
& \left.-k D \lambda_{w}^{-1}\left(e^{-\lambda_{w}\left(n-h_{m}\right)}-1\right)\right] \widetilde{U}_{I} .
\end{aligned}
$$

The constants $D$ and $E$ (constant with respect to the fast variables) are given by Eqs. (A3) and (A4) in the Appendix. In these equations,

$$
\gamma=\rho_{w} / \rho_{m}<1
$$

is the density ratio of the fluids,

$$
\zeta=\delta_{m} / \delta_{w}=\left(\nu_{m} / \nu_{w}\right)^{1 / 2}
$$

is the ratio of the Stokes boundary layer thicknesses, and

$$
\lambda_{f}=(1-i) / \delta_{f} \text { for } f=m, w
$$

is a complex parameter, in which

$$
\delta_{f}=\left(2 \nu_{f} / \sigma\right)^{1 / 2} \text { for } f=m, w
$$

is the Stokes boundary layer thickness for the respective fluid.

At $O(1)$, the interface kinematic boundary condition Eq. (14) reads

$$
\frac{\partial \xi}{\partial t_{1}}=v_{m 0} \quad \text { at } n=h_{m},
$$

which with Eq. (8) gives a relation for the amplitude of the interfacial waves

$$
b=\left.i \sigma^{-1} \widetilde{v}_{m}\right|_{n=h_{m}} .
$$

It is clear from this relation that the interfacial wave amplitude is indeed one order of magnitude smaller than that of the surface waves.

The near-bottom horizontal velocity $\tilde{U}_{I}$ and the dispersion relation, at the leading order, are obtainable from the inviscid theory: 


$$
\tilde{U}_{I}=\frac{\sigma a}{\sinh k h_{w}} \sim \frac{\sigma a}{\sinh k h_{0}}
$$

and

$$
\sigma^{2}=g k \tanh k h_{w} \sim g k \tanh k h_{0} .
$$

Since $\sigma$ and $a$ are given real constants and $h_{w}=h_{0}+O(\epsilon)$ is a constant at the leading order, then the near-bottom velocity $\tilde{U}_{I}$ and the wave number $k$ are also real constants at the leading order. See $\mathrm{Ng}^{11}$ for a further discussion on the kinematics in a two-layer Stokes boundary layer, including the wave attenuation rate and properties of the interfacial waves.

\section{B. Second-order steady currents}

With some algebra, one may obtain from Eq. (5) the $O(\epsilon)$ horizontal momentum equations, from which one may infer that the $O(\epsilon)$ velocities contain steady and temporal oscillating components. Taking a fast-time average of the velocities will remove the temporal oscillating components, and leave two steady components, one uniform and the other spatially periodic. Let us use an overbar to denote the time averaging over a wave period $T_{1}=2 \pi \sigma^{-1}$, i.e., for any function $A\left(t_{1}\right)$,

$$
\bar{A} \equiv \frac{1}{T_{1}} \int_{t_{1}}^{t_{1}+T_{1}} A d t_{1} .
$$

Taking the time averaging of the momentum equations followed by some mathematical manipulation then yields the following solutions:

$$
\begin{aligned}
& \text { In } 0 \leqslant \hat{n} \leqslant \hat{h}_{m}, \\
& \begin{aligned}
\bar{u}_{m 1}(\hat{n})= & k \sigma^{-1} \tilde{U}_{I}^{2}\left[\left(1-R^{2}\right) U_{m D}(\hat{n})+R \sin 2 \hat{x}_{1} U_{m A}(\hat{n})\right. \\
& +(1-\gamma) \sinh 2 k h_{0}\left(\frac{\partial \hat{h}_{m 0}}{\partial \hat{x}_{2}}+\frac{\partial \hat{h}_{m 1}}{\partial \hat{x}_{1}}\right) \\
& \left.\times \hat{n}\left(\frac{\hat{n}}{2}-\hat{h}_{m 0}\right)\right]
\end{aligned}
\end{aligned}
$$

and

$$
\begin{aligned}
\bar{v}_{m 1}(\hat{n})= & -k^{2} \delta_{m} \sigma^{-1} \widetilde{U}_{I}^{2}\left[2 R \cos 2 \hat{x}_{1} \int_{0}^{\hat{n}} U_{m A} d \hat{n}\right. \\
& \left.+(1-\gamma) \sinh 2 k h_{0} \frac{\partial^{2} \hat{h}_{m 1}}{\partial \hat{x}_{1}^{2}} \frac{\hat{n}^{2}}{2}\left(\frac{\hat{n}}{3}-\hat{h}_{m 0}\right)\right] .
\end{aligned}
$$

$$
\begin{aligned}
& \text { In } \hat{h}_{m} \leqslant \hat{n}<\infty, \\
& \begin{aligned}
\bar{u}_{w 1}(\hat{n})= & k \sigma^{-1} \widetilde{U}_{I}^{2}\left[\left(1-R^{2}\right) U_{w D}(\hat{n})+R \sin 2 \hat{x}_{1} U_{w A}(\hat{n})\right. \\
& \left.-(1-\gamma) \sinh 2 k h_{0}\left(\frac{\partial \hat{h}_{m 0}}{\partial \hat{x}_{2}}+\frac{\partial \hat{h}_{m 1}}{\partial \hat{x}_{1}}\right) \frac{\hat{h}_{m 0}^{2}}{2}\right]
\end{aligned}
\end{aligned}
$$

$$
\begin{aligned}
\bar{v}_{w 1}(\hat{n})= & -k^{2} \delta_{m} \sigma^{-1} \tilde{U}_{I}^{2}\left\{2 R \operatorname { c o s } 2 \hat { x } _ { 1 } \left(\int_{\hat{h}_{m}}^{\hat{n}_{w A}} U_{w A}\right.\right. \\
& \left.+\int_{0}^{\hat{h}_{m}} U_{m A} d \hat{n}\right)-(1-\gamma) \sinh 2 k h_{0} \\
& \left.\times \frac{\partial^{2} \hat{h}_{m 1}}{\partial \hat{x}_{1}^{2}} \frac{\hat{h}_{m 0}^{2}}{2}\left(\hat{n}-\frac{\hat{h}_{m 0}}{3}\right)\right\} .
\end{aligned}
$$

Recall that $\gamma=\rho_{w} / \rho_{m}$, and the normalized quantities (distinguished by a caret) have been defined in Table I. In the equations above, the functions $U_{m D}(\hat{n}), U_{m A}(\hat{n}), U_{w D}(\hat{n})$, and $U_{w A}(\hat{n})$ are nondimensional functions of the slow variables and their full-blown expressions are given in the Appendix.

The steady current in either layer of fluid is composed of Eulerian streaming and the density current; the former is due to viscosity in the boundary layer, while the latter results from a mild gradient of the water/liquid interface. The streaming itself comprises a uniform current, and an alternating current with a period of half-wavelength. For a dense liquid layer with a uniform thickness, the density current vanishes and the steady motion will solely be induced by streaming. The streaming current will be purely unidirectional when $R=0$, or purely alternating when $R=1$.

By integrating the horizontal velocity over the dense liquid layer followed by time averaging, we can get the net discharge rate of the dense liquid per unit width of wave crest:

$$
\begin{aligned}
Q= & \overline{\int_{0}^{h_{m}+\epsilon \xi} u_{m} d n} \\
= & \epsilon \int_{0}^{h_{m}} \bar{u}_{m 1} d n+\left.\epsilon \overline{\xi u_{m 0}}\right|_{n=h_{m}}+O\left(\epsilon^{2}\right) \\
= & k \delta_{m} \sigma^{-1} \widetilde{U}_{I}^{2}\left\{\left(1-R^{2}\right) Q_{D}+R \sin 2 \hat{x}_{1} Q_{A}\right. \\
& \left.-(1-\gamma) \sinh 2 k h_{0} \frac{\hat{h}_{m 0}^{3}}{3}\left(\frac{\partial \hat{h}_{m 0}}{\partial \hat{x}_{2}}+\frac{\partial \hat{h}_{m 1}}{\partial \hat{x}_{1}}\right)+O(\epsilon)\right\},
\end{aligned}
$$

where $Q_{D}$ and $Q_{A}$ are nondimensional functions of the slow variables given by

$$
Q_{D}=\int_{0}^{\hat{h}_{m}} U_{m D} d \hat{n}-\operatorname{Im}\left[\frac{1}{2} \widehat{\widetilde{v}}_{m} \hat{\tilde{u}}_{m}^{*}\right]_{\hat{n}=\hat{h}_{m}}
$$

and

$$
Q_{A}=\int_{0}^{\hat{h}_{m}} U_{m A} d \hat{n}+\operatorname{Re}\left[\hat{\widetilde{v}}_{m} \hat{\tilde{u}}_{m}^{*}\right]_{\hat{n}=\hat{h}_{m}} .
$$

The full-blown expressions for $Q_{D}$ and $Q_{A}$ are also given in the Appendix. The integral in the first step of Eq. (47) has been expanded in a Taylor series, thereby yielding the interface term. Note that $\epsilon$ has been inserted in the first two lines of Eq. (47), confirming that the net discharge is an $O(\epsilon)$ quantity. The steady wave streaming produces a uniform dis- 
charge that is in the direction of the incident wave and an alternating discharge that reverses in direction in half of a wavelength. The discharge due to gravity current, which occurs at the same time on the global and local scales, is in the direction of decreasing profile thickness on the corresponding scale.

The mass transport velocity, also called Lagrangian drift, which is the sum of Eulerian velocity and Stokes drift, ${ }^{7}$ is the time-averaged velocity of a fluid particle. The horizontal and vertical components of the mass transport velocity, $\left(u_{L}, v_{L}\right)$, can be found as follows:

$$
\begin{aligned}
u_{f L}(\hat{n})= & \bar{u}_{f 1}(\hat{n})+k \sigma^{-1} \tilde{U}_{I}^{2}\left\{-\left(1-R^{2}\right) \operatorname{Im}\left[\frac{1}{2} \frac{\partial}{\partial \hat{n}}\left(\hat{\widetilde{v}}_{f} \hat{\tilde{u}}_{f}^{*}\right)\right]\right. \\
& \left.+R \sin 2 \hat{x}_{1} \operatorname{Re}\left[\frac{\partial}{\partial \hat{n}}\left(\hat{\tilde{v}}_{f} \hat{\widetilde{u}}_{f}^{*}\right)\right]\right\}, \\
v_{f L}(\hat{n})= & \bar{v}_{f 1}(\hat{n})-k^{2} \delta_{m} \sigma^{-1} \tilde{U}_{I}^{2}\left\{2 R \cos 2 \hat{x}_{1} \operatorname{Re}\left[\hat{\tilde{v}}_{f} \hat{\tilde{u}}_{f}^{*}\right]\right\},
\end{aligned}
$$

where $0 \leqslant \hat{n} \leqslant \hat{h}_{m}$ for $f=m$, and $\hat{h}_{m} \leqslant \hat{n}<\infty$ for $f=w$. Further discussion on the mass transport velocity is presented in Sec. IV.

\section{Profile evolution}

Thus far, the solutions have been given in terms of the depth of the dense layer $h_{m}$, which varies slowly in time $\left(t_{2}\right.$ or longer) on the local $\left(x_{1}\right)$ and global $\left(x_{2}\right)$ length scales. In order to close the problem, we need to develop evolution equations for $h_{m}$ in terms of these variables. By a local scale, we mean a scale that is of the order of a wavelength, or physically, tens of meters. The global scale refers to the horizontal extent of the dense liquid layer, which is much longer than a wavelength and can be in the order of hundreds of meters.

Substituting Eq. (26) into the continuity equation (4) for $f=m$, which is then depth-integrated over the layer ( $\epsilon$ 's are again inserted for identification of order of magnitude):

$$
\int_{0}^{h_{m}+\epsilon \xi}\left[\frac{\partial u_{m}}{\partial x_{1}}+\epsilon \frac{\partial u_{m}}{\partial x_{2}}+\frac{\partial v_{m}}{\partial n}\right] d n=0 .
$$

Applying Leibnitz's rule and then the fast-time averaging, while using the boundary conditions (7) and (14) and the expansions (24)-(28), will give

$$
\begin{aligned}
& \epsilon \frac{\partial Q}{\partial x_{1}}+\epsilon^{2} \frac{\partial Q}{\partial x_{2}}+\frac{\partial h_{m 0}}{\partial t_{2}}+\epsilon \frac{\partial h_{m 1}}{\partial t_{2}}+\epsilon \frac{\partial h_{m 0}}{\partial t_{3}}+\epsilon^{2} \frac{\partial h_{m 0}}{\partial t_{4}} \\
& +O\left(\epsilon^{3}\right)=0,
\end{aligned}
$$

where the net discharge rate $Q$, an $O(\epsilon)$ quantity, is given by Eq. (47).

We further suppose that the water/liquid interface is initially flat on the local scale (i.e., $h_{m 1}=0$ at $t=0$ ). The only excitation on this scale is sinusoidally periodic, and in response the evolution of $h_{m 1}$ should also follow the same periodic dependence on $x_{1}$ with a zero spatial mean at all times:

$$
\left\langle h_{m 1}\right\rangle \equiv \frac{1}{L} \int_{0}^{L} h_{m 1} d x_{1}=0, \quad t>0 .
$$

Angle brackets are used to denote a local spatial average over one wavelength. Applying this average to Eq. (53), the equation reduces to

$$
\epsilon^{2} \frac{\partial\langle Q\rangle}{\partial x_{2}}+\frac{\partial h_{m 0}}{\partial t_{2}}+\epsilon \frac{\partial h_{m 0}}{\partial t_{3}}+\epsilon^{2} \frac{\partial h_{m 0}}{\partial t_{4}}+O\left(\epsilon^{3}\right)=0 .
$$

On collecting terms of the same order from Eqs. (53) and (55), one can arrive at the following equations:

$$
\begin{aligned}
& \frac{\partial h_{m 0}}{\partial t_{2}}=\frac{\partial h_{m 0}}{\partial t_{3}}=0, \\
& \frac{\partial Q}{\partial x_{1}}+\frac{\partial h_{m 1}}{\partial t_{2}}=0,
\end{aligned}
$$

and

$$
\frac{\partial\langle Q\rangle}{\partial x_{2}}+\frac{\partial h_{m 0}}{\partial t_{4}}=0 .
$$

Equations (57) and (58) are the two desired evolution equations that govern the profile change on the scales of $\left(x_{1}, t_{2}\right)$ and $\left(x_{2}, t_{4}\right)$, respectively. It is now apparent that the local displacements are purely spatially periodic and become zero when averaged on the global scale. On the global evolution, $h_{m 0}$ does not depend on $t_{2}$ and $t_{3}$, and it takes a much longer time $T_{4} \sim \epsilon^{-3} T_{1}$ for an appreciable change in the dense liquid profile to result from the slow spatial variation of the discharge rate. If the wave period is in the order of seconds, the spreading or migration of the bottom liquid may take days for significant changes.

When $R=1$ (i.e., pure standing waves), the wave streaming will not contribute to the global spreading of the dense liquid. On the other hand, when $R=0$ (i.e., pure progressive waves), local features will not evolve. For an intermediate $0<R<1$, local structures are formed on the time scale of $T_{2}$, while being conveyed by the global current which propagates on the time scale of $T_{4}$. These phenomena are further investigated in the next sections.

\section{UNDULATING INTERFACE ON THE LOCAL SCALE}

Substituting Eq. (47) into Eq. (57), we get a linear differential equation

$$
\frac{\partial \hat{h}_{m 1}}{\partial \hat{t}_{2}}-\frac{2(1-\gamma) \hat{h}_{m 0}^{3}}{3 \tanh k h_{0}} \frac{\partial^{2} \hat{h}_{m 1}}{\partial \hat{x}_{1}^{2}}=-R \cos 2 \hat{x}_{1} \frac{2 Q_{A}}{\sinh ^{2} k h_{0}},
$$

where $Q_{A}$ is given by Eq. (49) and fully detailed in the Appendix, and the normalized variables defined in Table I have been used. With the initial condition $\left.\hat{h}_{m 1}\right|_{\hat{t}_{2}=0}=0$, Eq. (59) can readily be solved to give

$$
\hat{h}_{m 1}\left(\hat{x}_{1}, \hat{t}_{2}\right)=H \cos 2 \hat{x}_{1}\left[1-\exp \left(-\alpha \hat{t}_{2}\right)\right],
$$

where

$$
H=-\frac{3 R Q_{A}}{2(1-\gamma) \hat{h}_{m 0}^{3} \sinh 2 k h_{0}}
$$



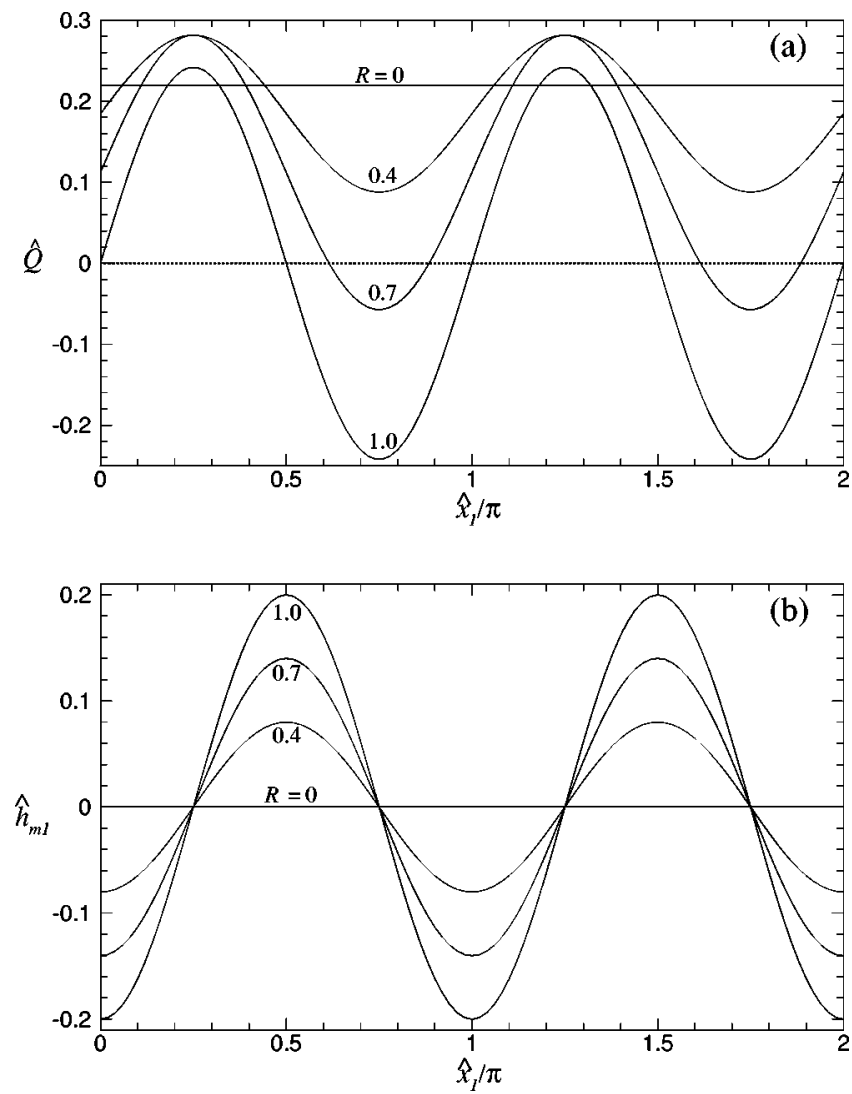

FIG. 2. Local longitudinal profiles of (a) initial discharge $\hat{Q}\left(\hat{x}_{1}\right)$, and (b) equilibrium undulating interface $\hat{h}_{m 1}\left(\hat{x}_{1}\right)$, as functions of the reflection coefficient $R$, where $\gamma=0.5$, and $\zeta=k h_{0}=\hat{h}_{m 0}=1.0$.

and

$$
\alpha=\frac{8(1-\gamma) \hat{h}_{m 0}^{3}}{3 \tanh k h_{0}} .
$$

Clearly, at a sufficiently large $\hat{t}_{2}$, the following equilibrium form of undulation will be developed on the local scale asymptotically:

$$
\hat{h}_{m 1} \rightarrow H \cos 2 \hat{x}_{1} \text { as } \hat{t}_{2} \gg 1 \text {. }
$$

Therefore, $H$ is the amplitude of a fully developed undulating water/liquid interface, and $\alpha$ specifies the rate at which this asymptotic state can be approached. Typically, the undulations are fully developed virtually within $\hat{t}_{2} \sim 5$. On reaching this stage, the components of discharge on the local scale due to wave streaming and gravity will exactly balance each other; hence the stage can be called a local equilibrium stage. One should not be confused with this interface undulation $h_{m 1}\left(x_{1}, t_{2}\right)$ and the oscillatory interface displacement $\xi\left(x_{1}, t_{1}\right)$; the former is a nonpropagating structure that results from mass transport and becomes permanent (as viewed on the local scale, and a time scale no longer than $T_{2}$ ) when fully developed, while the latter consists of propagating modes that oscillate with time on the fast scales without net mass transport.

The initial discharge $\hat{Q}\left(\hat{x}_{1}\right) \equiv Q /\left(k \delta_{m} \sigma^{-1} \tilde{U}_{I}^{2}\right)$ when $\hat{h}_{m 1}=0$ is shown in Fig. 2(a) as a function of the reflection coefficient $R$ for $\gamma=0.5$ and $h_{m 0}=\zeta=k h_{0}=1$. In this case,

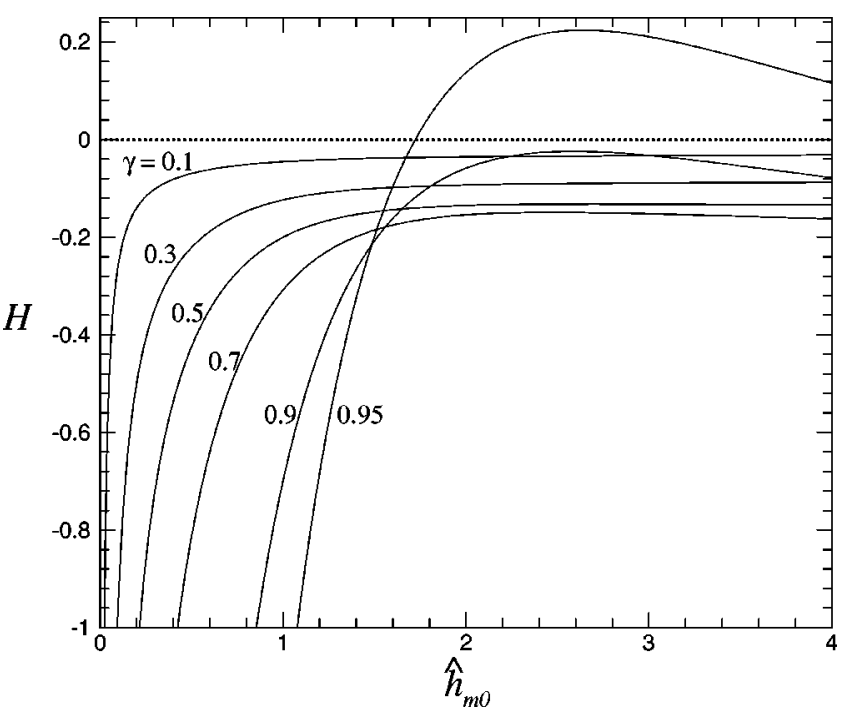

FIG. 3. Effects of the density ratio $\gamma$ and the thickness of the dense liquid layer $\hat{h}_{m 0}$ on the undulation amplitude $H$, where $\zeta=k h_{0}=R=1.0$.

the alternating component of the discharge converges toward the nodes $\left(\hat{x}_{1}=\pi / 2,3 \pi / 2, \ldots\right)$, and diverges away at the antinodes $\left(\hat{x}_{1}=0, \pi, 2 \pi, \ldots\right)$. As time increases, this alternating component of discharge will eventually die out when the undulation on the interface becomes fully developed, as given by Eq. (63) and shown in Fig. 2(b). The crests and troughs of the undulation are formed under the nodes and antinodes of the surface waves, respectively.

The undulation amplitude $H$ under a pure standing wave $(R=1)$ is examined in Fig. 3 as a function of $\gamma$ and $\hat{h}_{m 0}$. It is clear from this figure that the amplitude increases dramatically with a decreasing thickness of the dense liquid layer. Mathematically one can verify from Eq. (61) that for $R>0$, $H$ tends to blow up as $\hat{h}_{m 0}$ approaches zero. It is because the gravity current decreases with $\hat{h}_{m 0}^{3}$, while the alternating streaming current $Q_{A}$ decreases only with $\hat{h}_{m 0}^{2}$. The thinner the layer, the less static pressure the fluid can offer to resist mobilization due to streaming. A steeper interface gradient is therefore required for a thinner layer in order to produce a gravity current that is strong enough to balance the streaming current. The time to reach such an equilibrium stage will also be longer. The present theory of course breaks down when $\hat{h}_{m 0}$ becomes too small, or when $H$ becomes too large.

The undulation becomes more marked when $\gamma$ is closer to unity, or the density contrast (and hence the effective gravity) is smaller. It is remarkable that when the wave is purely standing and the density contrast is sufficiently small $(\gamma>0.9$ in the present case), there exists a critical thickness of the dense liquid layer for which $H=0$, or the undulation will not be developed at all. This happens only in the particular case that the streaming current forms closed circulating cells within the dense liquid layer even when the interface is flat. When this critical thickness is exceeded, the amplitude $H$ will change in sign. By then, the crests of the undulation will be formed at the antinodes instead.

Let us examine in further detail the flow structures in the boundary layer. Also for $\gamma=0.5$ and $\hat{h}_{m 0}=\zeta=k h_{0}=1$, Fig. 4 

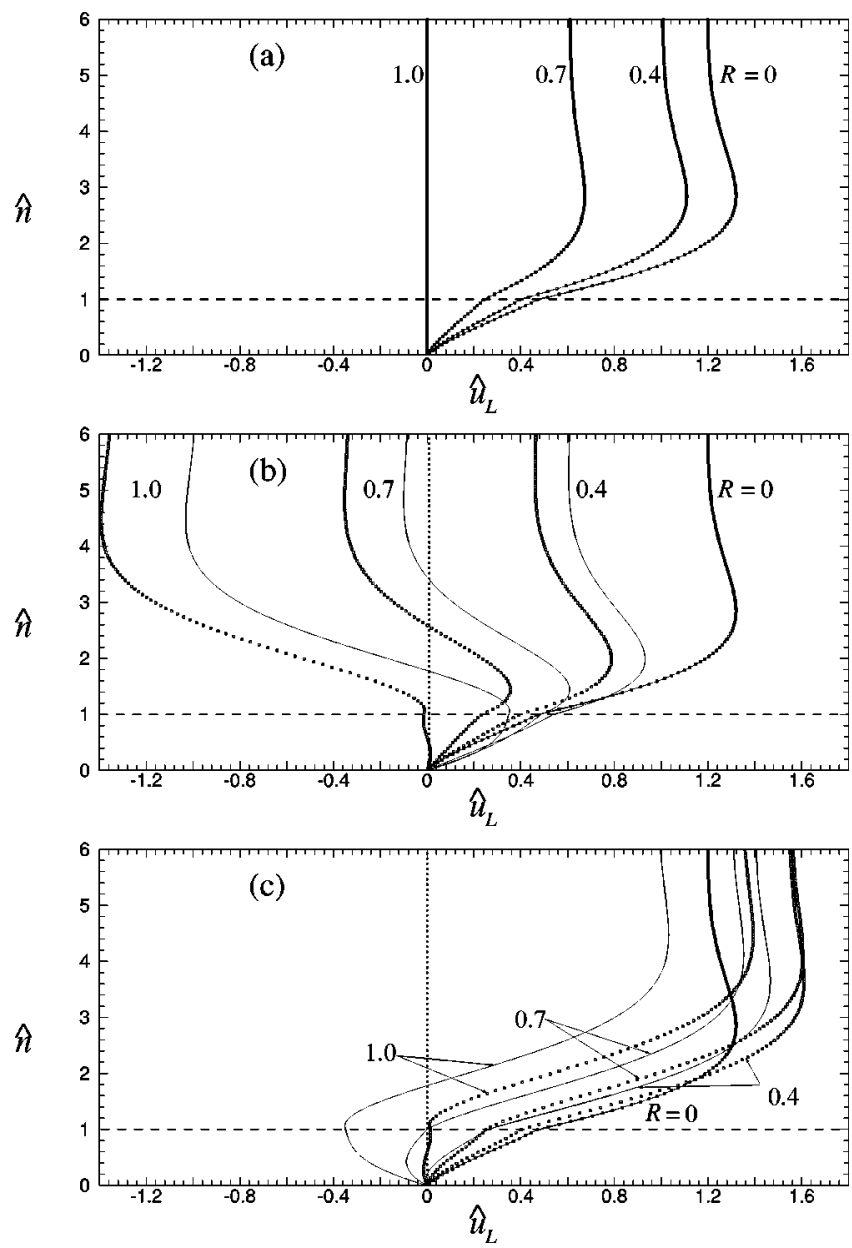

FIG. 4. Local vertical profiles of mass transport velocity $\hat{u}_{L}(\hat{n})$ in a twofluid Stokes boundary layer, as a function of the reflection coefficient $R$ at the positions (a) $\hat{x}_{1}=0$, (b) $\hat{x}_{1}=\pi / 4$, and (c) $\hat{x}_{1}=3 \pi / 4$, where $\gamma=0.5$, and $\zeta=k h_{0}=\hat{h}_{m 0}=1.0$. Solid lines are the initial profiles at $\hat{t}_{2}=0$, while squares denote the equilibrium profiles as $\hat{t}_{2} \gg 1$. The dashed line at $\hat{n}=1.0$ denotes the water/liquid interface.

shows the horizontal mass transport velocity component $\hat{u}_{L}$ $\equiv u_{L} /\left(k \sigma^{-1} \widetilde{U}_{I}^{2}\right)$ at three positions: (a) $\hat{x}_{1}=0$, (b) $\hat{x}_{1}=\pi / 4$, and (c) $\hat{x}_{1}=3 \pi / 4$, before (solid lines) and after (squares) the undulating interface is developed. The undulation, whose amplitude is one order of magnitude smaller than the layer thickness itself, is, however, not shown along the interface, which is simply denoted by a horizontal dashed line at $\hat{n}$ $=1$. Recall that above and below the interface are water and the dense liquid, respectively. The gravity current in the dense liquid layer is zero initially when the interface is flat, and flows from a crest (e.g., $\hat{x}_{1}=\pi / 2$ ) to a trough (e.g., $\hat{x}_{1}$ $=0, \pi)$ when the undulating interface is being developed. It is clear from Fig. 4 that Lagrangian drift of either fluid is essentially unidirectional (i.e., along the incident wave) in all stages when the reflection coefficient is relatively small $(R$ $=0,0.4)$. The flow structure becomes more complicated when the wave reflection turns out to be more substantial $(R=0.7,1.0)$. The profile of $\hat{u}_{L}$ then depends on $R, \hat{x}_{1}$, as well as the stage of the undulation development. Of particular interest are the points where the mass transport velocity reverses in direction, as they may correspond to the centers
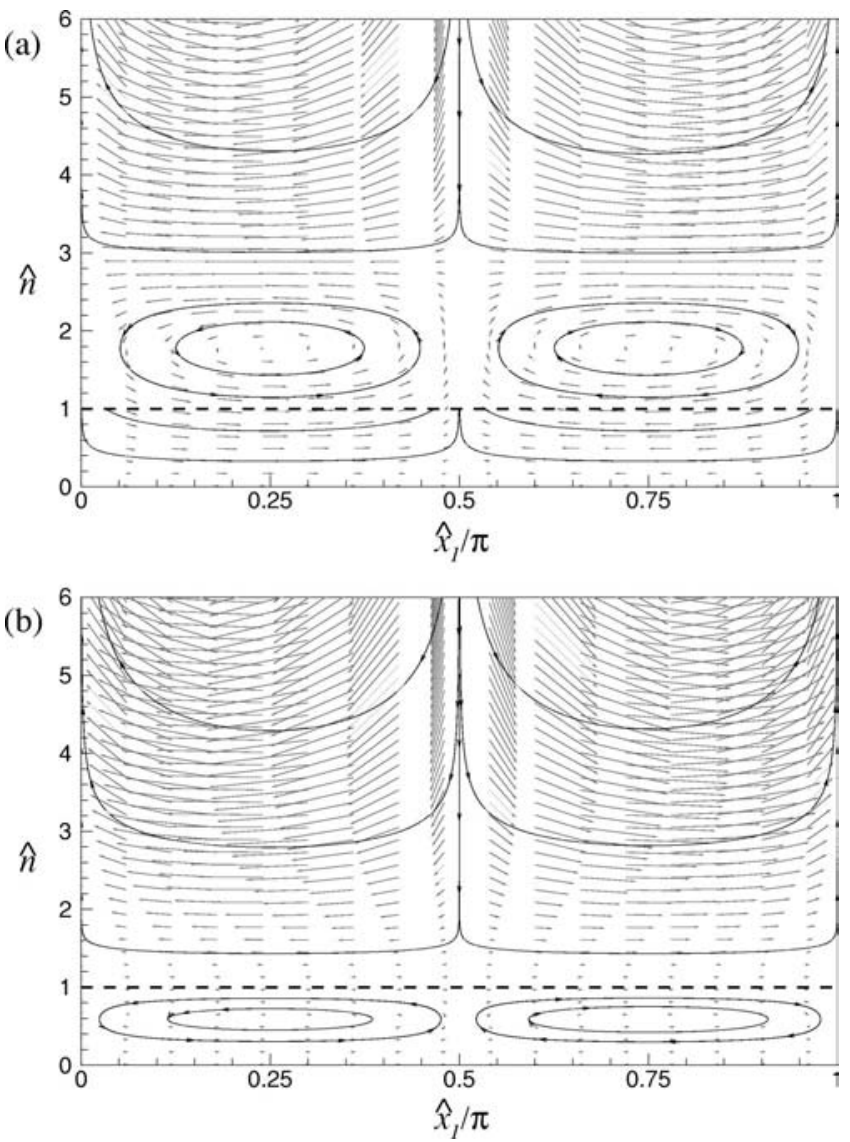

FIG. 5. Two-dimensional plots of mass transport velocity vectors and streamlines for the (a) initial stage, and (b) equilibrium stage, corresponding to the case of pure standing wave $R=1.0$ presented in Fig. 4 . The dashed line at $\hat{n}=1.0$ denotes the water/liquid interface.

of circulating cells. Such features are important for the suspension and accumulation of particles in the benthic boundary layer.

The two-dimensional flow patterns for $R=1$ and $R$ $=0.7$ are detailed in Figs. 5 and 6, which show the mass transport velocity vectors $\left(\hat{u}_{L}, \hat{v}_{L}\right)$ and the streamlines over a period equal to half-wavelength. Note that the vertical velocity component and the vertical coordinate have been stretched by the same factor, $\left(k \delta_{m}\right)^{-1}$, relative to their horizontal counterparts. Under a pure standing wave (Fig. 5), the mass transport in the top part of the water boundary layer (say, $\hat{n}>3$ ) is qualitatively the same as that in a single-fluid Stokes boundary layer. Namely, the fluid converges toward the antinodes, and diverges away from the nodes. ${ }^{8}$ Initially when the interface is flat, there are closed circulating cells in water above the interface, while the streaming current in the dense liquid always converges toward the nodes [Fig. 5(a)]. Such motion of the fluids leads to the formation of undulation crests at the nodes and troughs at the antinodes, as seen in Fig. 2. When the undulating interface is fully developed [Fig. 5(b)], closed circulating cells of a rather weak current are then developed in the dense liquid layer, consistent with the fact that the net discharge in the layer is now zero. Meanwhile on the water side, those original closed circulating cells above the interface are now completely suppressed, and as a result, the mass transport velocity farther up gains 

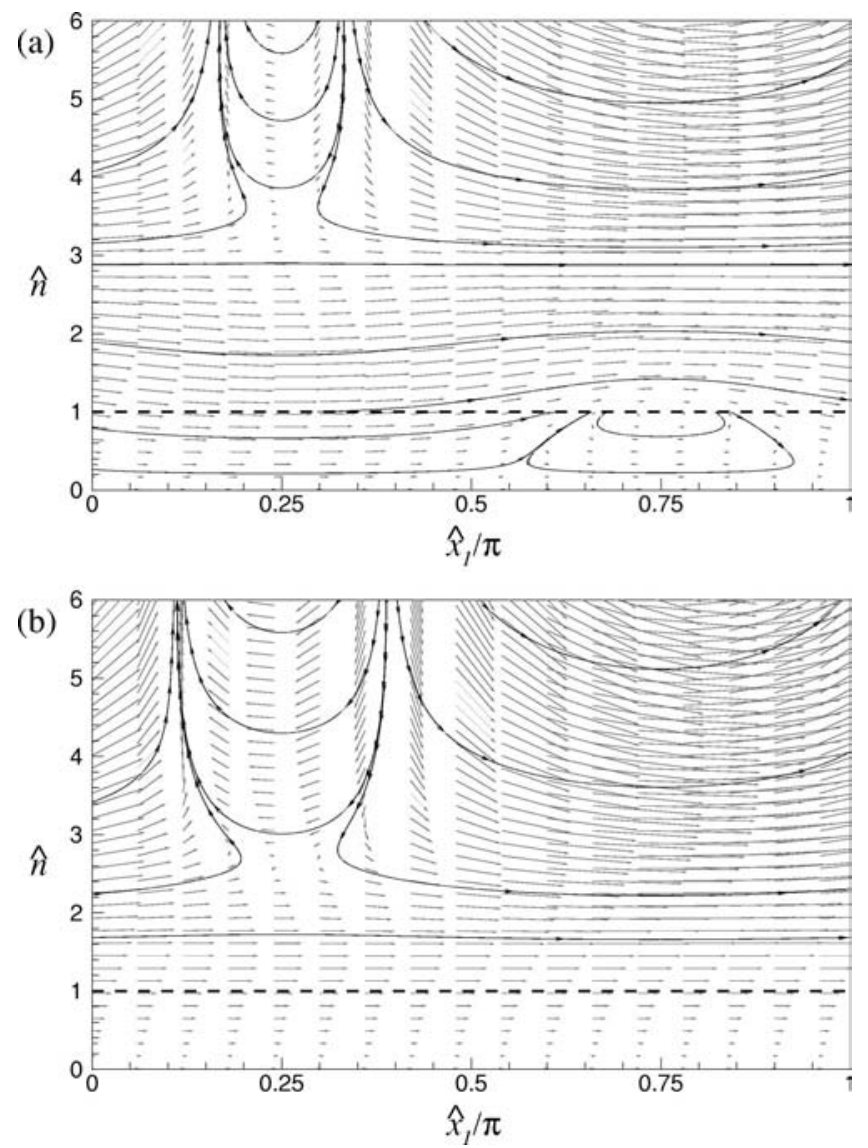

FIG. 6. Two-dimensional plots of mass transport velocity vectors and streamlines for the (a) initial stage, and (b) equilibrium stage, corresponding to the case of partial standing wave $R=0.7$ presented in Fig. 4 . The dashed line at $\hat{n}=1.0$ denotes the water/liquid interface.

strength and is greater in magnitude than it is initially. Water simply sinks at the nodes and escapes upward at the antinodes. The corresponding pictures are qualitatively different for a partial reflection, $R=0.7$ (Fig. 6). Owing to net steady currents, closed circulating cells do not exist in either fluid in either the initial or equilibrium stages. The initial flow nevertheless reverses in direction in a region centered at $\hat{x}_{1}$ $=\pi / 4$ in the top part of the water boundary layer, as well as in a region centered at $\hat{x}_{1}=3 \pi / 4$ in the dense liquid layer. As the equilibrium stage is approached, the flow reversal in water is enhanced and intensified, thereby leading to a reduced net mass flux of water longitudinally. On the other hand, the flow reversal in the dense liquid disappears entirely, resulting in a uniform steady discharge along the layer.

$\mathrm{Fu}^{14}$ has further studied other effects on the mass transport due to the local development of undulation. By and large, one may conclude from the present findings that the gravity current can be instrumental in regulating the local flow structures, and therefore the mass transport in a twofluid Stokes boundary layer can be materially different from that of a single fluid.

\section{SPREADING ON THE GLOBAL SCALE}

Let us now shift our focus to the global-scale balance between gravity and viscosity. Substituting Eq. (47) into Eq. (58), we may get a dimensionless governing equation for $\hat{h}_{m 0}\left(\hat{x}_{2}, \hat{t}_{4}\right)$ :

$$
\frac{\partial \hat{h}_{m 0}}{\partial \hat{t}_{4}}-\frac{1-\gamma}{6 \tanh k h_{0}} \frac{\partial^{2} \hat{h}_{m 0}^{4}}{\partial \hat{x}_{2}^{2}}=-\frac{1-R^{2}}{\sinh ^{2} k h_{0}} \frac{\partial Q_{D}}{\partial \hat{x}_{2}},
$$

where $Q_{D}$ is given by Eq. (48) and fully detailed in the Appendix, and the normalized time $\hat{t}_{4}$ is defined in Table I. The forcing term is proportional to the gradient of the unidirectional discharge rate $Q_{D}$, which is a function of the slow variables via dependence on $\hat{h}_{m 0}$. This term represents the net spreading effect due to streaming induced by surface waves.

The right-hand side of Eq. (64) vanishes if the surface wave is absent $\left(Q_{D}=0\right)$, or is a pure standing wave $(R$ $=1)$. In such cases, the spreading of the dense liquid will be driven solely by the gravity current. In dimensional form, Eq. (64) without the streaming term reduces to that obtained by Huppert [Ref. 4, Eq. (2.9)] for the propagation of a twodimensional viscous gravity current:

$$
\frac{\partial h_{m}}{\partial t}-\frac{(1-\gamma) g}{3 \nu_{m}} \frac{\partial}{\partial x}\left(h_{m}^{3} \frac{\partial h_{m}}{\partial x}\right)=0 .
$$

As reasoned earlier, our theory covers flow under a buoyancy-viscous balance, and serves as an extension of Huppert ${ }^{4}$ when the wave-induced streaming becomes as significant as the buoyancy in driving the flow.

If the total volume of the viscous fluid $q$ is finite and invariant with time and the spreading profile is symmetrical about $x=0$, an analytical similarity solution to Eq. (65) is available as follows: ${ }^{4,15}$

$$
\begin{aligned}
h_{m}(x, t)= & 0.842\left[\frac{3 q^{2} \nu_{m}}{(1-\gamma) g t}\right]^{1 / 5}\left(1-Y^{2}\right)^{1 / 3} \\
& \text { for }-x_{N} \leqslant x \leqslant x_{N},
\end{aligned}
$$

where

$$
Y(x, t)=0.709\left[\frac{(1-\gamma) g q^{3} t}{3 \nu_{m}}\right]^{-1 / 5} x
$$

and $x_{N}(t)$, which is the position of the leading front of the current, is given by $Y\left(x_{N}, t\right)=1$. For comparison with the present work, the solution above may be expressed in dimensionless form as follows, which is the solution to Eq. (64) without the forcing term:

$$
\begin{aligned}
\hat{h}_{m}\left(\hat{x}_{2}, \hat{t}_{4}\right)= & 0.842\left[\frac{3 \hat{q}^{2} \tanh k h_{0}}{2(1-\gamma) \hat{t}_{4}}\right]^{1 / 5}\left(1-Y^{2}\right)^{1 / 3} \\
& \text { for }-\hat{x}_{N} \leqslant \hat{x}_{2} \leqslant \hat{x}_{N},
\end{aligned}
$$

where

$$
Y\left(\hat{x}_{2}, \hat{t}_{4}\right)=0.709\left[\frac{2(1-\gamma) \hat{q}^{3} \hat{t}_{4}}{3 \tanh k h_{0}}\right]^{-1 / 5} \hat{x}_{2}
$$

and

$$
\hat{q}=q\left(k a / \delta_{m}\right)^{2}
$$

is the normalized total volume of the dense liquid.

Equation (64) is a nonlinear partial differential equation. Given an initial profile $\hat{h}_{m}\left(\hat{x}_{2}, 0\right)$, one may solve numerically the equation for $\hat{h}_{m}$ as a function of space and time. Figures 7-10 show the results for pure progressive waves (i.e., $R$ 


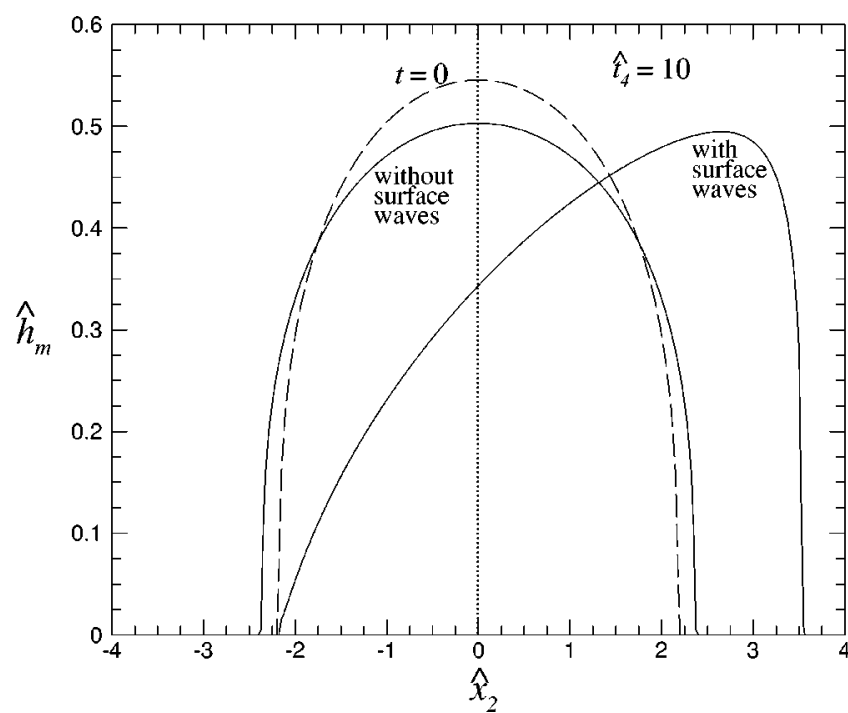

FIG. 7. Comparison between the longitudinal global profiles of the dense liquid $\hat{h}_{m}\left(\hat{x}_{2}, \hat{t}_{4}\right)$ spreading with and without surface waves at $\hat{t}_{4}=10.0$, where $R=0, \gamma=0.5$, and $\zeta=k h_{0}=1.0$. The dashed line is the initial profile obtained from Eq. (68) by setting $\hat{q}=1.0$ and $\hat{t}_{4}=20.0$.

$=0)$ that have been generated with a second-order explicit scheme of forward-time, centered-space finite-difference approximation of Eq. (64).

The initial profile in Fig. 7 is obtained from Eq. (68) with $\hat{q}=k h_{0}=1, \gamma=0.5$, and $\hat{t}_{4}=20$. The effects of surface waves are seen by comparing the profiles (a) without waves and (b) with waves $(\zeta=1.0)$, after a subsequent time interval of $\hat{t}_{4}=10$. Profile (a) is also obtainable from the analytical solution Eq. (68), and has been checked in agreement with that obtained independently by the numerical method. Without surface waves, the dense liquid continues to spread out symmetrically about the center $x=0$, while the maximum height of the layer keeps on decreasing. In the presence of surface waves, the streaming current enhances the flow in the

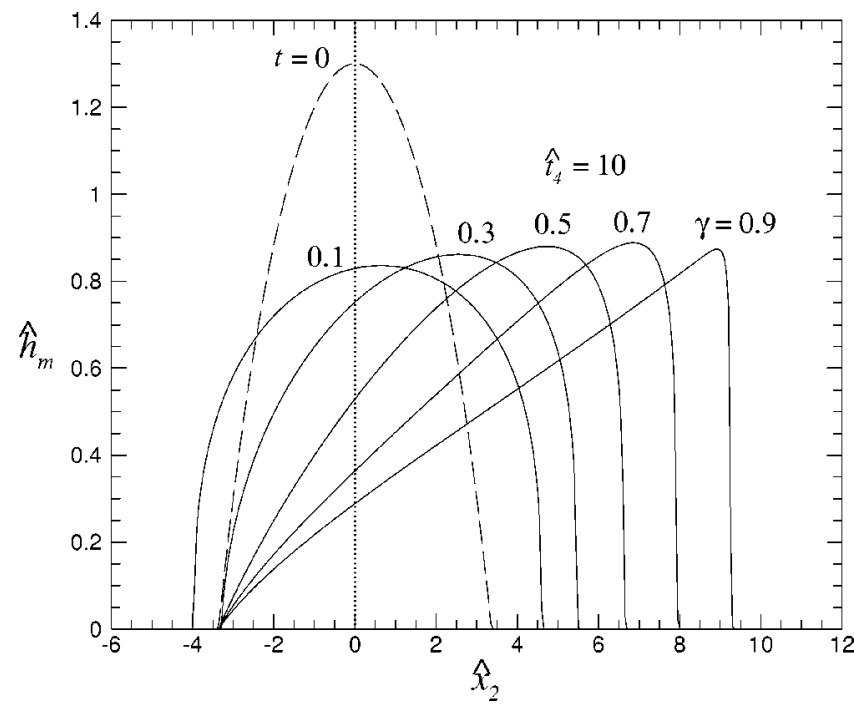

FIG. 8. Effects of the density ratio $\gamma$ on the global spreading profile of the dense liquid $\hat{h}_{m}\left(\hat{x}_{2}, \hat{t}_{4}\right)$ at $\hat{t}_{4}=10.0$, where $R=0$, and $\zeta=k h_{0}=1.0$. The dashed line is the initial profile.

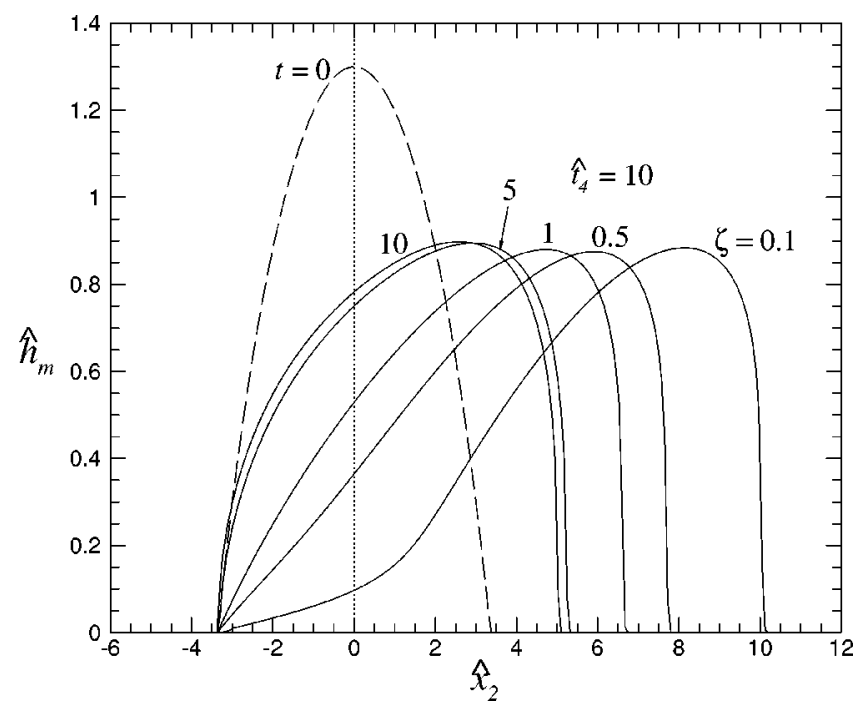

FIG. 9. Effects of the Stokes boundary layer thickness ratio $\zeta$ on the global spreading profile of the dense liquid $\hat{h}_{m}\left(\hat{x}_{2}, \hat{t}_{4}\right)$ at $\hat{t}_{4}=10.0$, where $R=0$, $\gamma=0.5$, and $k h_{0}=1.0$. The dashed line is the initial profile.

fore front, but counteracts the gravity current in the rear front. As a result, the entire dense liquid migrates in the direction of wave propagation; the center of mass of the profile is shifting forward gradually. While profiles (a) and (b) have approximately the same maximum height, the latter is clearly asymmetrical about its center of distribution; the front is steeper at the fore than the aft.

Effects of the ratios of density and viscosity and the water depth are shown in Figs. 8-10. In these cases, the initial profile is of a lens shape geometrically given by a segment of a circle. As shown in $\mathrm{Ng},{ }^{11}$ the discharge rate due to streaming decreases with $\gamma$ because a denser fluid tends to be more sluggish under wave forcing. This accounts for the different speeds of migration of the dense liquid for different values of the density ratio, shown in Fig. 8. The fore front, where the streaming and gravity currents add up positively, propagates the fastest and is therefore the steepest in the case of $\gamma=0.9$ when the liquid is only slightly heavier than water. The buoyancy force, which is relatively large for $\gamma \leqslant 0.3$,

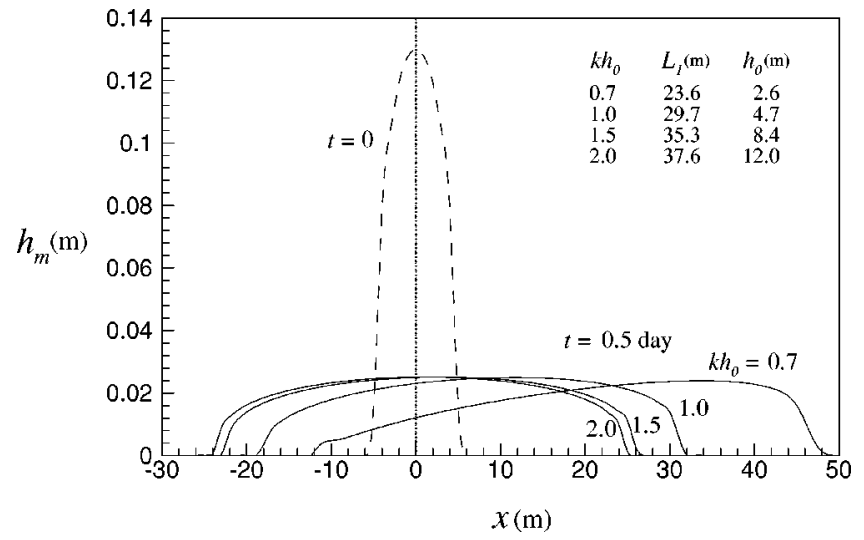

FIG. 10. Effects of the water depth $h_{0}$ on the global spreading profile of the dense liquid $h_{m}(x, t)$ at $t=0.5$ day, where $R=0, \gamma=0.5, \zeta=1.0, T_{1}=5 \mathrm{~s}$, and $a=\delta_{m}=0.1 \mathrm{~m}$. The dashed line is the initial profile. 
turns out to be subdominant in driving the propagation of the fore front. This is reasonable because the density current is proportional to the third power $\hat{h}_{m}$ [cf. Eq. (47)], which drops rapidly with the dense liquid layer thickness. The rear front, where the gravity and streaming currents negate each other, however, remains virtually stationary with time in most cases, although the profile gradient at this front is steeper for a smaller density ratio (greater buoyancy). The front even spreads rearward when the fluid is dense enough $(\gamma=0.1)$. As a result of the features occurring at the two fronts, the spreading profiles for $\gamma \geqslant 0.5$ are highly skewed toward their fore fronts (hence their peaks are sharper and closely behind the abrupt fore fronts), while those for $\gamma=0.1$, 0.3 have more rounded and symmetrical distributions about their peaks. The gravity current may outweigh the streaming current at the rear front for a sufficiently dense liquid.

The viscosity ratio $\zeta$ plays a similar role as $\gamma$ in controlling the spreading pattern of the dense liquid layer. The streaming velocity is larger for a smaller $\zeta$, resulting from a higher rate of momentum transfer across the water boundary layer. ${ }^{16}$ The streaming effect is reflected in Fig. 9, again, from the movement speed of the fore front, which clearly is higher for a smaller $\zeta$. Also, while the fore front moves forward with time, the rear front hardly moves in most cases. The profiles for $\zeta=5$ and 10, however, do not differ from each other appreciably. This is expected because, for one thing, the density current does not depend on this parameter, and the other, the wave streaming becomes rather insensitive to the viscosity ratio when the latter is larger than unity. ${ }^{11}$

As reasoned above, the migration of the dense liquid can be much controlled by the streaming current, which in turn depends on the influence of surface waves on the bottom boundary layer. Figure 10 shows the effects of the water depth $h_{0}$, via the dimensionless parameter $k h_{0}$, on the migration pattern of the layer. Since the normalization scales for the variables depend on $k$ which in turn varies with $h_{0}$, the present comparison is meaningful only in terms of the physical variables. In this plot, we consider a typical windwave period $T_{1}=5 \mathrm{~s}$, and an equal value for wave amplitude and Stokes boundary layer thickness $a=\delta_{m}=0.1 \mathrm{~m}$, and the spreading has lasted for a period of $t=0.5$ day. Other inputs are $R=0, \gamma=0.5$, and $\zeta=1.0$. The values of wavelength $L_{1}$ and water depth $h_{0}$ corresponding to each $k h_{0}$ are given in the figure. It is very clear that in shallow water $\left(k h_{0}=0.7\right)$ the wave streaming dominates over the buoyancy, while in deep water $\left(k h_{0}=2.0\right)$ the opposite is true. The streaming velocity is proportional to the square of the near-bottom inviscid velocity $\tilde{U}_{I}$, given by Eq. (40), and therefore decreases dramatically as $k h_{0}$ increases. In the case of $k h_{0}$ $=0.7$, the span of the layer has stretched from $10 \mathrm{~m}$ to more than $60 \mathrm{~m}$ in half a day, and the profile has turned to a highly skewed distribution, as seen in previous plots. We may infer from this plot that for typical wind-wave periods of $5 \mathrm{~s}$ and a wave amplitude $a=O(0.1 \mathrm{~m})$, a water depth of approximately $5 \mathrm{~m}$ can be the upper limit for an appreciable effect of the streaming current. This limiting water depth of course can be deeper for higher waves.

\section{CONCLUDING REMARKS}

An asymptotic theory is developed for the slow spreading of a thin layer of highly viscous immiscible dense liquid on the bottom of a near-shore waterway under the combined effects of surface waves and gravity current. The flow, excited by surface waves and driven by gravity, features several phenomena that become appreciable at different length and time scales.

The primary oscillatory motion occurs over a wave period, which is too short to see any net mass transport. For a sufficiently long time and in the presence of reflected waves, the alternating component of the streaming current can induce the development of an undulating interface between the near-bottom water and the dense liquid. Despite the small amplitude, the undulation in its course of formation can dramatically change the local structure of the mass transport velocity of the near-bottom water. The position and number of circulating cells above and below the interface are found to be dependent on the thickness of the dense liquid layer. A stratified wave boundary layer differs in nontrivial manners from a homogeneous one. It is worthwhile as a future extension of the present work looking into the much thicker secondary wave boundary layer, which is induced by convective inertia, when subject to the presence of a thin layer of dense viscous fluid on the bottom.

The global spreading of the dense liquid takes place on length and time scales longer than the wavelength and wave period. The streaming induced by progressive surface waves drives the dense liquid to migrate with a distinct pattern in the direction of wave propagation. The layer profile is always steeper at the fore than the aft, approaching a jump profile in the front while leaving a long tail at the back as time goes on. It is remarkable that the wave streaming is particularly influential under a relatively shallow water environment. Therefore the present theory is relevant to the transport of benthic matter in a long shallow nearshore waterway or navigation channel.

It will be desirable if experimental verification of the present theory, particularly Eq. (64), can be carried out. We, however, stress that our work has focused on the combined effects of two well-known mechanisms: streaming induced by surface waves and gravity current, which individually have been well backed by experiments. Therefore, the present work should offer a sound basis for a quantitative understanding of a streaming-buoyancy combined action on the propagation of a viscous current.

Of course, a more comprehensive model may be required in order to take into account other factors such as the bottom topography and tidal currents that are also influential in reality. Extensions of the present theory will be pursued in the future. A study on the spreading of a non-Newtonian dense fluid is underway and will be reported soon.

\section{ACKNOWLEDGMENTS}

The work was jointly supported by two grants from the Research Grants Council of the Hong Kong Special Admin- 
istrative Region, China (Project Nos. HKU 7117/99E and NSFC/HKU 8), and a CRCG Research Grant No. 10203302 awarded by the University of Hong Kong.

\section{APPENDIX: EXPRESSIONS FOR FUNCTIONS AND CONSTANTS IN THE STREAMING VELOCITIES AND DISCHARGE RATE}

Let us recall the streaming velocities, which are given by Eqs. (43) and (45). They are composed of direct and alternating currents that can be written in terms of functions and constants to be presented below:

$$
\begin{aligned}
& U_{m D}(\hat{n})=F_{m D}(\hat{n})+C_{m D} \hat{n}, \\
& U_{m A}(\hat{n})=F_{m A}(\hat{n})+C_{m A} \hat{n}, \\
& U_{w D}(\hat{n})=F_{w D}(\hat{n})+C_{w D}, \quad U_{w A}(\hat{n})=F_{w A}(\hat{n})+C_{w A},
\end{aligned}
$$

where the subscripts $D$ and $A$ denote the direct and alternating currents, respectively. Also recall that Re and Im denote, respectively, taking the real and imaginary parts, $i$ is the imaginary unit and the asterisk represents the complex conjugate.

We first define two constants $D$ and $E$ as follows:

$$
D=\frac{-\gamma \zeta-(1-\gamma) \zeta \cosh \lambda_{m} h_{m}}{\zeta \cosh \lambda_{m} h_{m}+\gamma \sinh \lambda_{m} h_{m}}
$$

and

$$
E=\frac{\gamma(1-\gamma)+\gamma^{2} \cosh \lambda_{m} h_{m}+\gamma \zeta \sinh \lambda_{m} h_{m}}{\zeta \cosh \lambda_{m} h_{m}+\gamma \sinh \lambda_{m} h_{m}} .
$$

For the current in the dense liquid, the real functions $F_{m D}$ and $F_{m A}$ are expressible as follows:

$$
F_{m D}(\hat{n})=\frac{1}{2} \operatorname{Re}\left[F_{m b}(\hat{n})-F_{m b}(0)\right]
$$

and

$$
F_{m A}(\hat{n})=F_{m a}(\hat{n})-F_{m a}(0)+\operatorname{Im}\left[F_{m b}(\hat{n})-F_{m b}(0)\right],
$$

where $F_{m a}(\hat{n})$ is a real function and $F_{m b}(\hat{n})$ is a complex function given by

$$
\begin{aligned}
F_{m a}(\hat{n})= & \gamma(\gamma-1) \hat{n}^{2}+2 \operatorname{Re}\left[i \gamma^{2} \cosh (1+i) \hat{n}\right. \\
& +i \gamma E \sinh (1-i) \hat{n}]+\frac{\gamma^{2}}{4}(\cosh 2 \hat{n}-\cos 2 \hat{n}) \\
& +\frac{|E|^{2}}{4}(\cosh 2 \hat{n}+\cos 2 \hat{n}) \\
& -\frac{\gamma}{2} \operatorname{Re}[E(\sinh 2 \hat{n}+\sinh 2 i \hat{n})]
\end{aligned}
$$

and

$$
\begin{aligned}
F_{m b}(\hat{n})= & \gamma^{2}[(1+i) \hat{n} \sinh (1+i) \hat{n}-2 \cosh (1+i) \hat{n}] \\
& -E^{*} \gamma[(1+i) \hat{n} \cosh (1+i) \hat{n}-2 \sinh (1+i) \hat{n}] \\
& +\frac{\gamma^{2}}{4}(\cosh 2 \hat{n}+\cosh 2 i \hat{n}) \\
& -\frac{\gamma E}{4}(\sinh 2 \hat{n}-\sinh 2 i \hat{n})+\frac{|E|^{2}}{4}(\cosh 2 \hat{n} \\
& -\cosh 2 i \hat{n})-\frac{E^{*} \gamma}{4}(\sinh 2 \hat{n}+\sinh 2 i \hat{n}) \\
& -i E \gamma \sinh (1+i) \hat{n}+i|E|^{2} \cosh (1+i) \hat{n} . \quad \text { (A8) }
\end{aligned}
$$

The real constants $C_{m D}$ and $C_{m A}$, which are constant with respect to the fast coordinates, are expressible as

$$
C_{m D}=\frac{1}{2} \operatorname{Re}\left[\left(-\frac{\partial F_{m b}}{\partial \hat{n}}+\frac{\gamma}{\zeta^{2}} \frac{\partial F_{w b}}{\partial \hat{n}}\right)_{\hat{n}=\hat{h}_{m 0}}-C_{2 a}\right]
$$

and

$$
\begin{aligned}
C_{m A}= & \left(-\frac{\partial F_{m a}}{\partial \hat{n}}+\frac{\gamma}{\zeta^{2}} \frac{\partial F_{w a}}{\partial \hat{n}}\right)_{\hat{n}=\hat{h}_{m 0}} \\
& +\operatorname{Im}\left[\left(-\frac{\partial F_{m b}}{\partial \hat{n}}+\frac{\gamma}{\zeta^{2}} \frac{\partial F_{w b}}{\partial \hat{n}}\right)_{\hat{n}=\hat{h}_{m 0}}-C_{2 a}\right],
\end{aligned}
$$

in which the derivatives of the functions are given by

$$
\begin{aligned}
\frac{\partial F_{m a}}{\partial \hat{n}}= & 2 \gamma(\gamma-1) \hat{h}_{m 0}+2 \operatorname{Re}\left[(-1+i) \gamma^{2} \sinh (1+i) \hat{h}_{m 0}+(1+i) \gamma E \cosh (1-i) \hat{h}_{m 0}\right]+\frac{\gamma^{2}}{2}\left(\sinh 2 \hat{h}_{m 0}+\sin 2 \hat{h}_{m 0}\right) \\
& +\frac{|E|^{2}}{2}\left(\sinh 2 \hat{h}_{m 0}-\sin 2 \hat{h}_{m 0}\right)-\gamma \operatorname{Re}\left[E\left(\cosh 2 \hat{h}_{m 0}+i \cosh 2 i \hat{h}_{m 0}\right)\right], \\
\frac{\partial F_{m b}}{\partial \hat{n}}= & \gamma^{2}\left[2 i \hat{h}_{m 0} \cosh (1+i) \hat{h}_{m 0}\right]-E^{*} \gamma\left[2 i \hat{h}_{m 0} \sinh (1+i) \hat{h}_{m 0}\right]+\frac{\gamma^{2}}{2}\left(\sinh 2 \hat{h}_{m 0}+i \sinh 2 i \hat{h}_{m 0}\right) \\
& -\frac{\gamma E}{2}\left(\cosh 2 \hat{h}_{m 0}-i \cosh 2 i \hat{h}_{m 0}\right)+\frac{|E|^{2}}{2}\left(\sinh 2 \hat{h}_{m 0}-i \sinh 2 i \hat{h}_{m 0}\right)-\frac{E^{*} \gamma}{2}\left(\cosh 2 \hat{h}_{m 0}+i \cosh 2 i \hat{h}_{m 0}\right) \\
& +(1-i) E \gamma \cosh (1+i) \hat{h}_{m 0}-(1-i)|E|^{2} \sinh (1+i) \hat{h}_{m 0}-\gamma^{2}(1+i) \sinh (1+i) \hat{h}_{m 0}+E^{*} \gamma(1+i) \cosh (1+i) \hat{h}_{m 0}, \\
\frac{\partial F_{w a}}{\partial \hat{n}}= & 2 \operatorname{Re}[-(1+i) D \zeta]-|D|^{2} \zeta,
\end{aligned}
$$


and

$$
\begin{aligned}
\frac{\partial F_{w b}}{\partial \hat{n}}= & (1-i) \zeta^{2} D^{*}\left\{\gamma\left[(1-i) \hat{h}_{m 0}-\sinh (1-i) \hat{h}_{m 0}\right]\right. \\
& \left.+E\left[\cosh (1-i) \hat{h}_{m 0}-1\right]\right\}-(1+i) \zeta D^{*}\left[\hat{h}_{m 0} \zeta(1\right. \\
& +i)+1]+i \zeta\left(2 \zeta D^{*} \hat{h}_{m 0}-|D|^{2}\right),
\end{aligned}
$$

and the complex constant $C_{2 a}$ is

$$
\begin{aligned}
C_{2 a}= & (1+i)\left\{\gamma\left[(1+i) \hat{h}_{m 0}-i \sinh (1-i) \hat{h}_{m 0}\right]\right. \\
& \left.+i E\left[\cosh (1-i) \hat{h}_{m 0}-1\right]\right\} \\
& \times\left[-\gamma \cosh (1+i) \hat{h}_{m 0}+E^{*} \sinh (1+i) \hat{h}_{m 0}-\gamma D^{*}\right] .
\end{aligned}
$$

Similarly, for the current in water, the real functions $F_{w D}(\hat{n})$ and $F_{w A}(\hat{n})$ can be expressed as

$$
F_{w D}(\hat{n})=\frac{1}{2} \operatorname{Re}\left[F_{w b}(\hat{n})\right]
$$

and

$$
F_{w A}(\hat{n})=F_{w a}(\hat{n})+\operatorname{Im}\left[F_{w b}(\hat{n})\right],
$$

where the real function $F_{w a}(\hat{n})$ and the complex function $F_{w b}(\hat{n})$ are given by

$$
F_{w a}(\hat{n})=2 \operatorname{Re}\left[i D e^{-(1-i) \zeta\left(\hat{n}-\hat{h}_{m 0}\right)}\right]+\frac{|D|^{2}}{2} e^{-2 \zeta\left(\hat{n}-\hat{h}_{m 0}\right)}
$$

and

$$
\begin{aligned}
F_{w b}(\hat{n})= & i \zeta\left\{\gamma\left[(1-i) \hat{h}_{m 0}-\sinh (1-i) \hat{h}_{m 0}\right]\right. \\
& \left.+E\left[\cosh (1-i) \hat{h}_{m 0}-1\right]\right\} D^{*} e^{-(1+i) \zeta\left(\hat{n}-\hat{h}_{m 0}\right)} \\
& +D^{*}[(1+i) \zeta \hat{n}+2] e^{-(1+i) \zeta\left(\hat{n}-\hat{h}_{m 0}\right)} \\
& -(1+i) \zeta \hat{h}_{m 0} D^{*} e^{-(1+i) \zeta\left(\hat{n}-\hat{h}_{m 0}\right)} \\
& +\frac{|D|^{2}}{2} e^{-2 \zeta\left(\hat{n}-\hat{h}_{m 0}\right)}+i|D|^{2} e^{-(1+i) \zeta\left(\hat{n}-\hat{h}_{m 0}\right)} .
\end{aligned}
$$

The real constants $C_{w D}$ and $C_{w A}$ are

$$
C_{w D}=\left(F_{m D}-F_{w D}\right)_{\hat{n}=\hat{h}_{m 0}}+C_{m D} \hat{h}_{m 0}+\frac{1}{2} \operatorname{Re}\left[C_{1 a}\right]
$$

and

$$
C_{w A}=\left(F_{m A}-F_{w A}\right)_{\hat{n}=\hat{h}_{m 0}}+C_{m A} \hat{h}_{m 0}+\operatorname{Im}\left[C_{1 a}\right],
$$

in which the complex constant $C_{1 a}$ is

$$
\begin{aligned}
C_{1 a}= & \left\{\gamma\left[(1+i) \hat{h}_{m 0}-i \sinh (1-i) \hat{h}_{m 0}\right]\right. \\
& \left.+i E\left[\cosh (1-i) \hat{h}_{m 0}-1\right]\right\} \\
& \times\left[-\gamma \sinh (1+i) \hat{h}_{m 0}+E^{*} \cosh (1+i) \hat{h}_{m 0}+\zeta D^{*}\right] .
\end{aligned}
$$

The net discharge rate of the dense liquid, given by Eq. (47), also comprises of direct and alternating components:

$$
\begin{aligned}
Q_{D}= & \frac{1}{2}\left\{\operatorname{Re}\left[I F_{m b}\left(\hat{h}_{m 0}\right)\right]-\operatorname{Re}\left[I F_{m b}(0)\right]\right. \\
& \left.-\operatorname{Re}\left[F_{m b}(0)\right] \hat{h}_{m 0}+C_{m D} \hat{h}_{m 0}^{2}+\operatorname{Re}\left[F_{i n}\right]\right\}, \\
Q_{A}= & \left\{I F_{m a}\left(\hat{h}_{m 0}\right)+\operatorname{Im}\left[I F_{m b}\left(\hat{h}_{m 0}\right)\right]\right\}-\left\{I F_{m a}(0)\right. \\
& \left.+\operatorname{Im}\left[I F_{m b}(0)\right]\right\}-\left\{F_{m a}(0)+\operatorname{Im}\left[F_{m b}(0)\right]\right\} \hat{h}_{m 0} \\
& +C_{m A} \hat{h}_{m 0}^{2} / 2+\operatorname{Im}\left[F_{i n}\right],
\end{aligned}
$$

where

$$
\begin{aligned}
& I F_{m a}(\hat{n})=\gamma \operatorname{Re}[(1-i) \gamma \sinh (1-i) \hat{n}-(1-i) \\
& \left.\times E \cosh (1-i) \hat{n}-\frac{E}{4}(\cosh 2 \hat{n}-i \cos 2 \hat{n})\right] \\
& +\gamma(\gamma-1) \frac{\hat{n}^{3}}{3}+\frac{\gamma^{2}}{8}(\sinh 2 \hat{n}-\sin 2 \hat{n}) \\
& +\frac{|E|^{2}}{8}(\sinh 2 \hat{n}+\sin 2 \hat{n}), \\
& I F_{m b}(\hat{n})=\gamma^{2}\left[\hat{n} \cosh (1+i) \hat{n}-\frac{1-i}{2} \sinh (1+i) \hat{n}\right. \\
& -(1-i) \sinh (1+i) \hat{n}]-E^{*} \gamma[\hat{n} \sinh (1+i) \hat{n} \\
& \left.-\frac{1-i}{2} \cosh (1+i) \hat{n}-(1-i) \cosh (1+i) \hat{n}\right] \\
& +\frac{\gamma^{2}}{8}(\sinh 2 \hat{n}+\sin 2 \hat{n})-\frac{\gamma E}{8}(\cosh 2 \hat{n} \\
& +i \cos 2 \hat{n})+\frac{|E|^{2}}{8}(\sinh 2 \hat{n}-\sin 2 \hat{n}) \\
& -\frac{\gamma E^{*}}{8}(\cosh 2 \hat{n}-i \cos 2 \hat{n})-\frac{1+i}{2} \gamma E \\
& \times \cosh (1+i) \hat{n}+\frac{1+i}{2}|E|^{2} \sinh (1+i) \hat{n},
\end{aligned}
$$

and the complex interface term is expressed as

$$
\begin{aligned}
F_{i n}= & \frac{1+i}{2}\left\{\gamma\left[(1-i) \hat{h}_{m 0}-\sinh (1-i) \hat{h}_{m 0}\right]\right. \\
& \left.+E\left[\cosh (1-i) \hat{h}_{m 0}-1\right]\right\} \\
& \times\left[\gamma-\gamma \cosh (1+i) \hat{h}_{m 0}+E^{*} \sinh (1+i) \hat{h}_{m 0}\right] .
\end{aligned}
$$

${ }^{1}$ T. B. Benjamin, "Gravity currents and related phenomena," J. Fluid Mech. 31, 209 (1968).

${ }^{2}$ H. E. Huppert and J. E. Simpson, "The slumping of gravity currents," J. Fluid Mech. 99, 785 (1980).

${ }^{3} \mathrm{~N}$. Didden and T. Maxworthy, "The viscous spreading of plane and axisymmetric gravity currents," J. Fluid Mech. 121, 27 (1982).

${ }^{4} \mathrm{H}$. E. Huppert, "The propagation of two-dimensional and axisymmetric viscous gravity currents over a rigid horizontal surface," J. Fluid Mech.

121, 43 (1982). 
${ }^{5} \mathrm{M}$. Ungarish and H. E. Huppert, "The effects of rotation on axisymmetric gravity currents," J. Fluid Mech. 362, 17 (1998).

${ }^{6}$ A. J. Hogg, H. E. Huppert, and M. A. Hallworth, "Reversing buoyancy of particle-driven gravity currents," Phys. Fluids 11, 2891 (1999).

${ }^{7}$ M. S. Longuet-Higgins, "Mass transport in water waves," Philos. Trans. R. Soc. London, Ser. A 245, 535 (1953).

${ }^{8}$ T. G. Carter, P. L.-F. Liu, and C. C. Mei, "Mass transport by waves and offshore sand bedforms," J. Waterways, Harbors and Coastal Eng. Div. ASCE 99, 165 (1973).

${ }^{9}$ R. A. Dalrymple and P. L.-F. Liu, "Waves over soft muds: a two-layer fluid model," J. Phys. Oceanogr. 8, 1121 (1978).

${ }^{10}$ T. Sakakiyama and E. W. Bijker, "Mass transport velocity in mud layer due to progressive waves," J. Waterw., Port, Coastal, Ocean Eng. 115, 614 (1989).

${ }^{11}$ C. O. $\mathrm{Ng}$, "Water waves over a muddy bed: a two-layer Stokes' boundary layer model," Coastal Eng. 40, 221 (2000).

${ }^{12}$ C. C. Mei and K.-F. Liu, "A Bingham-plastic model for a muddy seabed under long waves," J. Geophys. Res. 92, 14581 (1987).

${ }^{13}$ C. C. Mei, The Applied Dynamics of Ocean Surface Waves (World Scientific, Singapore, 1989).

${ }^{14}$ S. C. Fu, M.Phil. thesis, The University of Hong Kong, Hong Kong, 2001.

${ }^{15}$ G. I. Barenblatt, Similarity, Self-similarity, and Intermediate Asymptotics (Plenum, New York, 1979).

${ }^{16} \mathrm{C}$. O. $\mathrm{Ng}$, "Mass transport in a two-layer wave boundary layer," Ocean Eng. 28, 1393 (2001). 